\title{
Instability of sintering of porous bodies
}

\author{
Eugene Olevsky ${ }^{\mathrm{a}, *}$, Alain Molinari ${ }^{\mathrm{b}}$ \\ ${ }^{a}$ San Diego State University, Mechanical Engineering Department, 5500 Campanile Drive, San Diego, \\ CA 92182-1323, USA \\ ${ }^{\mathrm{b}}$ Laboratoire de physique et médcanique des matériaux, I.S.G.M.P., Université de Metz, Ile du Saulcy, \\ 57045 Metz Cedex 1, France
}

Received in final revised form 27 May 1999

\begin{abstract}
Sintering models are discussed and used to analyze flow instabilities that may arise during preliminary compaction of powders. These instabilities can be at the origin of heterogeneities in the densification. The material is modeled as a viscoplastic thermal sensitive porous material. The modeling includes the limit case of a linear viscous material. The effects of sintering conditions (temperature and pressure in the case of pressure sintering) and the effects of material characteristics such as porosity, heat capacity, theoretical density, surface tension, particle size and creep parameters on stability of sintering are investigated. The heat release associated with the plastic flow is shown to sometimes have an important role. Stability criteria are derived and applied to the analysis of sintering and hot isostatic pressing, using various sintering models. These stability criteria can be used to optimize the densification process; one can control, for example, temperature so as to avoid any instability. Stability maps enabling an optimization of temperature-pressure regime in hot isostatic pressing are built for sample metal (nickel) powder. (C) 2000 Elsevier Science Ltd. All rights reserved.
\end{abstract}

Keywords: Sintering; Porous body; Stability; Perturbation

\section{Introduction}

The effect of localization of densification (Skorohod and Solonin, 1984) is one of the important technological problems related to sintering. It is known from sintering practice that, in some cases, an intense densification occurs in some parts of a porous body, whereas in other parts, large pores are formed. This effect is an evident

\footnotetext{
* Corresponding author. Fax: + 1-619-594-3599.
} 
manifestation of an instability caused by initial small inhomogeneities in the spatial distribution of pores. This phenomenon can lead to the various microstructure defects' nucleation, macroscopic warpage and damage. The sintering trajectory is influenced by small differences in initial density distribution or temperature heterogeneities. A non-uniform density distribution provoked by sintering instability can cause deficient mechanical properties of the final product. Sintering is generally incapable of compensating for defects or problems introduced in earlier processing steps (for example, density heterogeneiety due to the preliminary pressing). The understanding of the influences of these defects is a fundamental challenge. Little quantitative effort has been put into these areas (German, 1996, p. 508). The stability of sintering of thin films on rigid substrates has been investigated (Bordia and Jagota, 1993). This research work has provided an insight on the behavior of constrained sintering. However, the stability problems of free sintering and of isostatic pressing have not been considered in a general form. The present work is an attempt to investigate the effects of material properties and of loading conditions on the stability of sintering and an isostatic pressing process.

Many models of sintering have been proposed in the literature and no unified point of view seems to exist presently. However, beyond an apparent diversity, the models agree on some general trends. As the stability depends strongly on the characteristics of the material constitutive modeling, it is worth to discuss in some details the general framework adopted to model the material response during sintering.

Theoretical concepts of sintering were originally based upon ideas of a discrete organization of porous media. Sintering was considered as the collective result of thermally activated adhesion processes which produce the growth of contacts between particles and their coalescence. The analysis of the mechanisms of interparticle interaction was characteristic for many of the elaborated approaches in the theory of sintering. These approaches were directed toward the investigation of the local kinetics of the process. Important features of sintering kinetics, such as the contact formation and the nature of the driving force have been clarified (German, 1996). The results obtained have been embodied in a number of kinetic relationships describing densification under free sintering.

A different and promising approach is connected with the use of continuum mechanics, which has been successfully applied to the description of compaction of porous bodies. This approach, based upon the theories of plastic (Kuhn and Downey, 1971; Green, 1972; Shima and Oyane, 1976; Gurson, 1977; Corapcioglu and Uz, 1978; Shtern et al., 1982; Tvergaard, 1982; Doraivelu et al., 1984; Kim and Carroll, 1987; Fleck et al., 1992) and nonlinear-viscous deformation of porous bodies (Wilkinson and Ashby, 1975; Helle et al., 1985; Ponte Castaneda, 1991; Sofronis and McMeeking, 1992; Lee and Mear, 1992), can be designated as a continuum theory of sintering (Skorohod, 1972; Scherer, 1977; Druyanov and Vartanov, 1984; Bordia and Scherer, 1988; Olevsky and Skorohod, 1988; Skorohod et al., 1989; Jagota and Hui, 1990a, 1990b; Reid and Oakberg, 1990; Riedel, 1990; Skorohod et al., 1991; Du and Cocks, 1992a,b; McMeeking, 1992; Mori, 1992; Svoboda and Riedel, 1992; Sveboda et al., 1994; Cocks and Du, 1993; Olevsky and Skorohod, 
1993a,b,c; Riedel et al., 1994; Skorohod et al., 1993a,b,c; Cocks, 1994; Reid, 1994; Riedel and Svoboda, 1993; Olevsky, 1994; Zhao and Dharani, 1994; Cocks and Aparicio, 1995; Hong and Dharani, 1995; Svoboda and Riedel, 1995a, 1995b; Olevsky et al., 1996; Olevsky and Bert, 1997; Svoboda et al., 1996).

The paper is organized as follows. In Section 2, we present and discuss the general equations adopted here to model the free sintering of nonlinear-viscous materials. In Section 3, we analyze the stability of the process of free sintering and isostatic pressing by using a perturbation method. Such methods have been used to predict the instability of viscoplastic flow related to material or geometrical softening. For example, the process of adiabatic shear banding in metals subjected to high rates of strain can be analyzed in its initial stage by using perturbation methods as shown by Raiser and Clifton (1993) and Molinari et al. (1988) (Dudzinski and Molinari, 1991; Molinari et al., 1993).

It is demonstrated that stability of an isothermal sintering process depends mainly on the relationship between porosity and the material strain rate sensitivity. If the self-heating, due to the dissipation of part of the mechanical work into heat, is accounted for, it is shown that instability can be significantly favored for fast sintering process. Similar results are obtained for isostatic compaction. The stability criteria that we obtain can be used to control the pressure and temperature histories so as to avoid any instability during the compaction process.

\section{Phenomenological model of sintering}

The following is a formulation of a continuum theory of sintering of porous nonlinear-viscous materials. A porous medium is considered as a two-phase material made up of the substance ("porous body skeleton") and of voids (pores). The skeleton, in turn, can be a multiphase material. The skeleton is assumed to be made of individual particles having a nonlinear-viscous incompressible isotropic behavior. The voids (pores) are isotropically distributed. The overall response is therefore isotropic.

The free energy $F$ per unit mass of porous medium is, by hypothesis, a function of the absolute temperature $T$ and of the specific volume $\vartheta$

$$
F=F(T, \vartheta)
$$

with

$$
\vartheta=\frac{1}{\hat{\rho}}
$$

where $\hat{\rho}$ is the volumetric mass of porous material. The second law of thermodynamics can be expressed in terms of the Clausius-Duhern inequality, where heat conduction has been neglected: 


$$
\sigma_{i j}: \dot{\varepsilon}_{i j}-\hat{\rho} \dot{F}-\hat{\rho} S \dot{T} \geqslant 0
$$

$\sigma_{i j}$ and $\dot{\varepsilon}_{i j}$ are the Cauchy stress tensor and the strain rate tensor, respectively; $S$ is the entropy per unit mass, "." represents the material time derivative. The Einstein summation convention on repeated indeces is used here. Introducing (1) into (3), we obtain

$$
\left(\sigma_{i j}-\frac{\partial F}{\partial \vartheta} \delta_{i j}\right) \dot{\varepsilon}_{i j}-\hat{\rho}\left(\frac{\partial F}{\partial T}+S\right) \dot{T} \geqslant 0
$$

where $\delta_{i j}$ is the Kronecker symbol. In deriving the latter equation, we have used the following result:

$$
\frac{\dot{\vartheta}}{\vartheta}=-\frac{\dot{\hat{\rho}}}{\hat{\rho}}=\dot{\varepsilon}_{i j}
$$

The second part of (5) comes from mass conservation.

Note that, for a viscous material, $\sigma_{i j}$ and $S$ depend on the strain rate $\dot{\varepsilon}_{i j}$ but not on $\dot{T}$. Therefore, since (4) holds for any $\dot{T}$, we have:

$$
S=-\frac{\partial F}{\partial T}
$$

and (4) reduces to the following dissipation inequality:

$$
\left(\sigma_{i j}-P_{\mathrm{L}} \delta_{i j}\right) \dot{\varepsilon}_{i j} \geqslant 0
$$

where we have defined the effective Laplace pressure (sintering stress) by:

$$
P_{\mathrm{L}}=\left.\frac{\partial F}{\partial \vartheta}\right|_{\mathrm{T}}
$$

The condition (7) is in particular satisfied if there exists a dissipation potential $D$ defined as a homogeneous function of order $m+1$ of the strain rate $\dot{\varepsilon}_{i j}$ such that:

$$
\sigma_{i j}-P_{\mathrm{L}} \delta_{i j}=\frac{\partial D}{\partial \dot{\varepsilon}_{i j}}
$$

Then the Euler identity leads to:

$$
\dot{\varepsilon}_{i j} \frac{\partial D}{\partial \dot{\varepsilon}_{i j}}=(m+1) D \geqslant 0
$$

In general, $D$ is also a function of the specific volume $\vartheta$ or, equivalently, a function of the porosity $\theta$ defined as the ratio of the volume of pores and the total volume: 


$$
\theta=\frac{V_{\text {pores }}}{V_{\text {total }}}
$$

To have some understanding of the effect of the porosity on the overall response, we consider different model materials.

\subsection{Linear-viscous incompressible material with voids}

We consider first the case of a linear-viscous incompressible fluid containing isotropically distributed voids. The overall behavior is isotropic. The dissipation potential in this case is:

$$
D=\eta \gamma^{2}+\frac{1}{2} \zeta e^{2}
$$

where $\eta$ and $\zeta$ are the effective shear and bulk moduli. The following notations are used:

$$
\gamma=\sqrt{\dot{\varepsilon}_{i j}^{\prime} \dot{\varepsilon}_{i j}^{\prime}} \quad e=\operatorname{tr} \dot{\square}=\dot{\varepsilon}_{i i}
$$

where $\dot{\varepsilon}_{i j}^{\prime}$ is the deviator of the strain rate tensor, and $e$ is the shrinkage rate. In the Appendix, it is shown that (Skorohod, 1972):

$$
\eta=\varphi \eta_{o} \quad \zeta=2 \psi \eta_{o}
$$

with

$$
\varphi=(1-\theta)^{2} \quad \psi=\frac{2}{3} \frac{(1-\theta)^{3}}{\theta}
$$

$\eta$ and $\zeta$ are dependent on the shear modulus $\eta_{o}$ of the skeleton (incompressible fluid). Using (13), the potential $D$ can be expressed as:

$$
D=(1-\theta) \eta_{o} W^{2}
$$

with

$$
W=\sqrt{\frac{\varphi \gamma^{2}+\psi e^{2}}{1-\theta}}
$$

defining an equivalent strain rate.

From Hill's identity, the macroscopic dissipation potential $D$ is the volume average over the porous medium of the microscopic potential $d$. 


$$
D=\langle d\rangle
$$

Since $d=0$ in the pores, we have:

$$
D=(1-\theta)\langle d\rangle_{\text {skeleton }}=(1-\theta) \eta_{o}\left\langle\gamma_{s}^{2}\right\rangle_{\text {skeleton }}
$$

with $\gamma_{s}=\sqrt{\dot{\varepsilon}_{i j}^{s} \dot{\varepsilon}_{i j}^{s}}, \dot{\varepsilon}_{i j}^{s}$ being the microscopic deviatoric strain rate in the skeleton. From (15) it follows that:

$$
W=\sqrt{\left\langle\gamma_{s}^{2}\right\rangle_{\text {skeleton }}}
$$

The constitutive law is given by (9) and (15):

$$
\sigma_{i j}=2 \eta_{o}\left(\varphi \dot{\varepsilon}_{i j}^{\prime}+\psi e \delta_{i j}\right)+P_{\mathrm{L}} \delta_{i j}
$$

providing that

$$
\frac{\partial W}{\partial \dot{\varepsilon}_{i j}}=\frac{1}{(1-\theta) W}\left(\varphi \dot{\varepsilon}_{i j}^{\prime}+\psi e \delta_{i j}\right)
$$

From (18) we have:

$$
p=\frac{1}{3} \operatorname{tr} \sigma=2 \eta_{o} \psi e+P_{\mathrm{L}}
$$

Defining the second invariant of the deviatoric stress $\sigma_{i j}^{\prime}$ by

$$
\tau=\sqrt{\sigma_{i j}^{\prime} \sigma_{i j}^{\prime}}
$$

we obtain:

$$
\tau=2 \eta_{o} \varphi \gamma
$$

Substituting $e$ and $\gamma$ from (20), (22) into Eq. (16) results in the following relationship between the equivalent strain rate $W$ and equivalent stress $\sigma$ :

$$
\sigma=2 \eta_{o} W
$$

with $\sigma$ defined by:

$$
\sigma=\frac{1}{\sqrt{1-\theta}} \sqrt{\frac{\tau^{2}}{\varphi}+\frac{\left(P-P_{\mathrm{L}}\right)^{2}}{\psi}}
$$


For $\theta=0$, the material is incompressible. When $\theta \rightarrow 0$ we have $\varphi \rightarrow 1$, $\psi \rightarrow \infty, e \rightarrow 0$ and $\psi \mathrm{e}^{2} \rightarrow 0$. Therefore $W \rightarrow \gamma$ and the dissipation potential of a linear-viscous fluid is retrieved from (15):

$$
D=\eta_{o} \gamma^{2}
$$

\subsection{Nonlinear-viscous porous material}

Among the models used in this paper we shall present here the one proposed by Skorohod and Olevsky (Olevsky and Skorohod, 1988; Olevsky et al., 1996). This formulation is purely phenomenological although it relies on some micro-macro transition in the linear case.

A nonlinear-viscous porous material is considered. The skeleton is first assumed to have the behavior of a nonlinear-viscous incompressible fluid defined by the following dissipation potential:

$$
D_{\text {matrix }}=\frac{A}{m+1} \gamma_{s}^{m+1}
$$

where $\gamma_{s}$ is considered at the level of the skeleton.

It is postulated that the dissipation potential of the porous material has the form:

$$
D=\frac{A}{m+1}(1-\theta) W^{m+1}
$$

with $W$ defined in Eq. (16). We have seen that $W \rightarrow \gamma$ when $\theta \rightarrow 0$. Therefore, in the case of a nonporous material $(\theta=0)$, the dissipation potential (27) coincides with the expression (26). Note that, for $m=1$, the expression (27) reduces to the potential (15) corresponding to a porous linear-viscous material $\left(A=2 \eta_{o}\right)$.

Using Eqs. (9), (19) and (27), we obtain the following constitutive law:

$$
\sigma_{i j}=A W^{m-1}\left(\varphi \dot{\varepsilon}_{j i}^{\prime}+\psi e \delta_{i j}\right)+P_{\mathrm{L}} \delta_{i j}
$$

from which we have [see the definition $(21)$ of $\tau$ ]:

$$
\tau=A \varphi W^{m-1} \gamma \quad p=A \psi W^{m-1} e+P_{\mathrm{L}}
$$

Substituting into Eq. (16) the values of $\gamma$ and $e$ obtained from (29) leads to the following relationship between the equivalent stress $\sigma$ and the equivalent strain rate $W$ (defined in Eqs. (24) and (16), respectively]:

$$
\sigma=A W^{m}
$$


For $m \rightarrow 0$, the limiting case of a rigid perfectly plastic porous material is obtained with the yield limit defined by:

$$
\sigma=\frac{1}{\sqrt{1-\theta}} \sqrt{\frac{\tau^{2}}{\varphi}+\frac{\left(p-P_{\mathrm{L}}\right)^{2}}{\psi}} \leqslant A
$$

where $A / \sqrt{2}$ is the yield limit of the porous body skeleton in pure shear. Many different models have been formulated for plastic porous materials (Kuhn and Downey, 1971; Green, 1972; Shima and Oyane, 1976; Gurson, 1977; Corapcioglu and Uz, 1978; Shtern et al., 1982; Tvergaard, 1982; Doraivelu et al., 1984; Kim and Carroll, 1987; Fleck et al., 1992). A comparison can be made by considering the case where $P_{\mathrm{L}}$ is negligible. Assuming that the loading is purely hydrostatic, the yield pressure given by Eq. (31) can be expressed with use of Eq. (14) as:

$$
p=\sqrt{\frac{2}{3}} A \frac{(1-\theta)^{2}}{\sqrt{\theta}}=\frac{2}{3} \sigma_{0} \frac{(1-\theta)^{2}}{\sqrt{\theta}}
$$

where $\sigma_{0}=\sqrt{\frac{3}{2}} A$ is the yield stress of the matrix in simple tension. With the same hypothesis the Green (1972) and Gurson (1977) models predict, for small porosities, a yield pressure in the form:

$$
p=-\frac{2}{3} \sigma_{0} \ln \theta
$$

For the stability analysis, the trends are important. The experimental trends seem to favor the formulation (31) as well as other model approaches (Kuln and Downey, 1971; Shima and Oyane, 1976; Corapcioglu and Uz, 1978; Doraivelu et al., 1984; Kim and Carroll, 1987) which do not provide the logarithmic form (33) of the yield pressure for small porosities.

Finally, a more general framework can be proposed by assuming a general relationship between the equivalent stress and the equivalent strain rate:

$$
\sigma=\sigma(W)
$$

The constitutive law can be represented in the form:

$$
\sigma_{i j}=\frac{\sigma(W)}{W}\left(\varphi \dot{\varepsilon}_{i j}^{\prime}+\psi e \delta_{i j}\right)+P_{\mathrm{L}} \delta_{i j}
$$

The corresponding dissipation potential is:

$$
D=(1-\theta) \int_{0}^{W} \sigma\left(W^{\prime}\right) \mathrm{d} W^{\prime}
$$


The relationship (35) is an example of a basic constitutive expression used in the continuum theory of sintering. This formulation can be used for the description of a wide range of processes of powder treatment. $\sigma_{i j}$ corresponds to the macroscopic stress applied to a representative volume element. The first term of the right-hand part characterizes the material resistance, and the second term corresponds to the influence of capillary stresses (sintering factor). In the case where the macroscopic stress $\sigma_{i j}$ (respectively the Laplace pressure $P_{\mathrm{L}}$ ) is equal to zero, Eq. (35) describes free sintering (respectively treatment by pressure without sintering). In general, Eq. (35) describes sintering under pressure.

From Eq. (35) we have:

$$
\tau=\frac{\sigma(W)}{W} \varphi \gamma \quad p=\frac{\sigma(W)}{W} \psi e+P_{\mathrm{L}}
$$

From these equations one can obtain an important relationship between the invariants of stress and of strain rate:

$$
\left(p-P_{\mathrm{L}}\right) \varphi \gamma=\tau \psi e
$$

To complete the set of constitutive equations, for an isothermal problem, one has to specify the dependence of the Laplace pressure $P_{\mathrm{L}}$ upon the porosity $\theta$ and to consider the evolution law for $\theta$ :

$$
\frac{\dot{\theta}}{1-\theta}=e
$$

To account for thermal effects, it will be assumed that the equivalent stress is a function of the absolute temperature $T$ :

$$
\sigma=\sigma(W, T)
$$

The evolution law for the temperature is the heat equation:

$$
\rho c \dot{T}-k \Delta T=\beta\left(\sigma_{i j} \dot{\varepsilon}_{i j}-P_{\mathrm{L}} e\right)
$$

where $\rho, c$ and $k$ are the effective volumetric mass, heat capacity and heat conductivity, respectively. $\Delta T$ designates the Laplacian of the temperature. In the righthand side we have the heat source that corresponds to the part $\beta(0 \leqslant \beta \leqslant 1)$ of the rate of viscoplastic deformation work which is dissipated into heat. In the following we shall consider that $\beta=1$. Only adiabatic processes will be examined (see discussion below), thus the heat conduction term in (41) will be neglected.

The effective heat capacity is supposed to depend both on temperature and porosity as follows:

$$
c=c_{0}(T) \bar{c}(\theta)
$$


The volumetric mass $\rho$ has the form:

$$
\rho=\rho_{o}(1-\theta)
$$

where $\rho_{o}$ is the volumetric mass of the skeleton.

Using the expression (35) of the constitutive response, the energy equation (41) can be rewritten, in the case of an adiabatic process, and with $\beta=1$, as:

$$
\rho c \dot{T}=\frac{\sigma(W)}{W}\left(\varphi \gamma^{2}+\psi \mathrm{e}^{2}\right)
$$

To conclude that presentation, we emphasize that no attempt was made here to elaborate a rigorous micro-macro transition by which the overall behavior of the porous material would be determined from the constitutive behavior of the matrix, the volume fraction, shape and spatial distribution of pores. The model proposed coincides with a micro-macro model in the linear case but is purely phenomenological in the nonlinear case. The functions $\varphi(\theta)$ and $\psi(\theta)$ defined by Eq. (14) can be obtained in the framework of a micro-macro approach for a linear-viscous behavior. There is no theoretical evidence that they would keep exactly the same form for a nonlinear-viscous material. However, experimental results have confirmed the validity of the modeling adopted here for many practical applications (see, for example, Skorohod, 1972; Olevsky et al., 1997).

\section{Stability analysis}

A significant nonuniform distribution of the density is frequently observed after sintering. In this section, we shall explore cases where such nonuniformity could be the result of the development of an instability in the viscoplastic flow. Thermomechanical coupling might be a critical factor. To clarify the effects of the temperature dependence of the material response, we shall develop two approaches. The first ignores thermal effects, the problem is assumed to be isothermal. In the second analysis, thermomechanical coupling is fully accounted for.

The fundamental solution of the sintering problem is first considered. This solution corresponds to an ideal uniform distribution of porosity. The stability of this fundamental solution is then analyzed with the use of a perturbation approach.

\subsection{Isothermal problem}

We assume here that the temperature is uniform within the specimen and does not evolve with time:

$$
T=T_{o}=\text { const }
$$

Therefore, we shall not introduce an explicit temperature dependence in the governing equations. 
For free sintering $\left(\sigma_{i j}=0\right)$ it results from (35) that:

$$
\dot{\varepsilon}_{i j}^{\prime}=0
$$

and

$$
p=\frac{\sigma(w)}{W} \psi e+P_{\mathrm{L}}=0
$$

From (46) we have $\gamma=0$. From (16) and $e<0$, it follows that:

$$
W=-\sqrt{\frac{\psi}{1-\theta} e}
$$

The effective Laplace pressure is given by:

$$
P_{\mathrm{L}}=P_{\mathrm{L} o} \cdot \bar{P}_{\mathrm{L}}(\theta)
$$

$\bar{P}_{\mathrm{L}}(\theta)$ depends on the sintering model considered (see Table 1). $P_{\mathrm{L} o}=\frac{2 \alpha}{r_{o}}$ (see the Appendix), is constant under isothermal conditions. Note that $P_{\mathrm{L}}>0$.

The fundamental solution is obtained in the following way. Introducing in (47) the expressions (48) and (49) for $W$ and $P_{\mathrm{L}}$ and the expression for $\psi$ from Table 1 results in an implicit equation for $e$ in terms of $\theta$. The evolution of 0 is given by the differential equation (39).

For sintering processes, a linear-viscous response $[m=1$ in Eq. (30)] is in many cases relevant. The shrinkage rate $(e)$ can be then expressed with (47) [which reduces to Eq. (20)] as:

$$
e=-\frac{P_{\mathrm{L} o}}{2 \eta_{o}} \frac{\bar{P}_{\mathrm{L}}(\theta)}{\psi(v)}
$$

The expressions for the normalized Laplace pressure (sintering stress) $\bar{P}_{\mathrm{L}}(\theta)$ and for the normalized bulk viscosity $\psi(\theta)$ are given in Table 1 and represented in Figs. 1 and 2 for three models: Helle-Easterling-Ashby (Helle et al., 1985; Ashby, 1990), Scherer (Scherer, 1979; Bordia and Scherer, 1988) and Skorohod-Olevsky (Skorohod, 1972; Olevsky et al., 1996). Note that the Skorohod-Olevsky model provides values of $\psi(\theta)$ and $\bar{P}_{\mathrm{L}}(\theta)$ intermediate between the two other models. Note also the irregular behavior predicted by the Helle et al. model for the value of porosity $\theta=0.08$. The corresponding evolutions of the porosity in terms of the normalized time:

$$
t_{s}=\frac{P_{\mathrm{L} o}}{\eta_{o}} t
$$

(where $t$ is the physical time) are shown in Fig. 3 . 


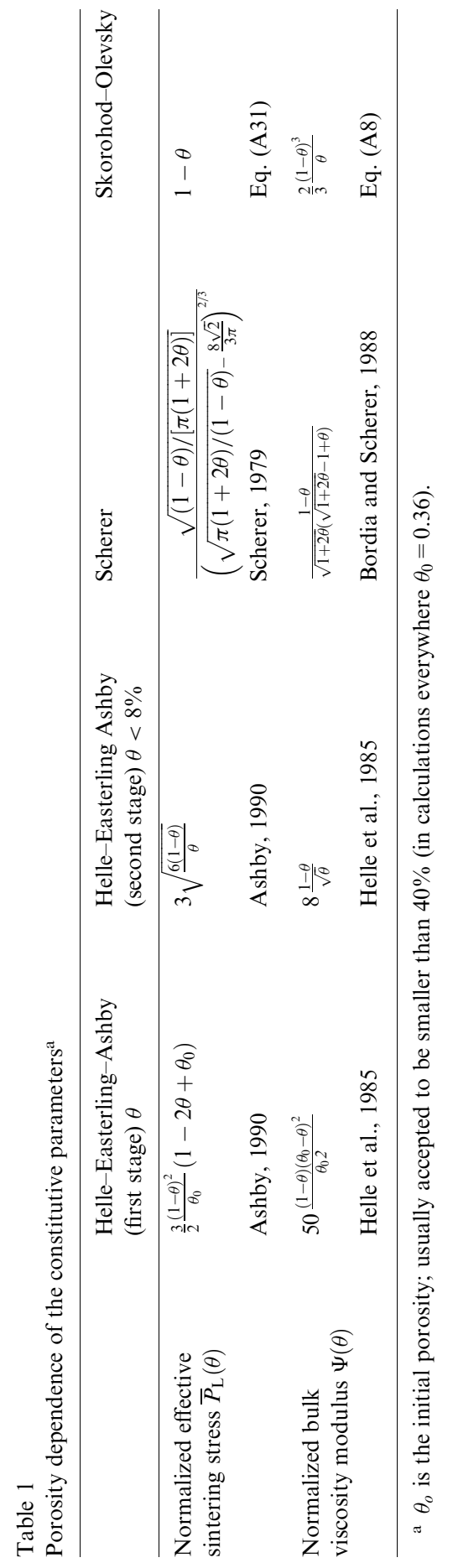




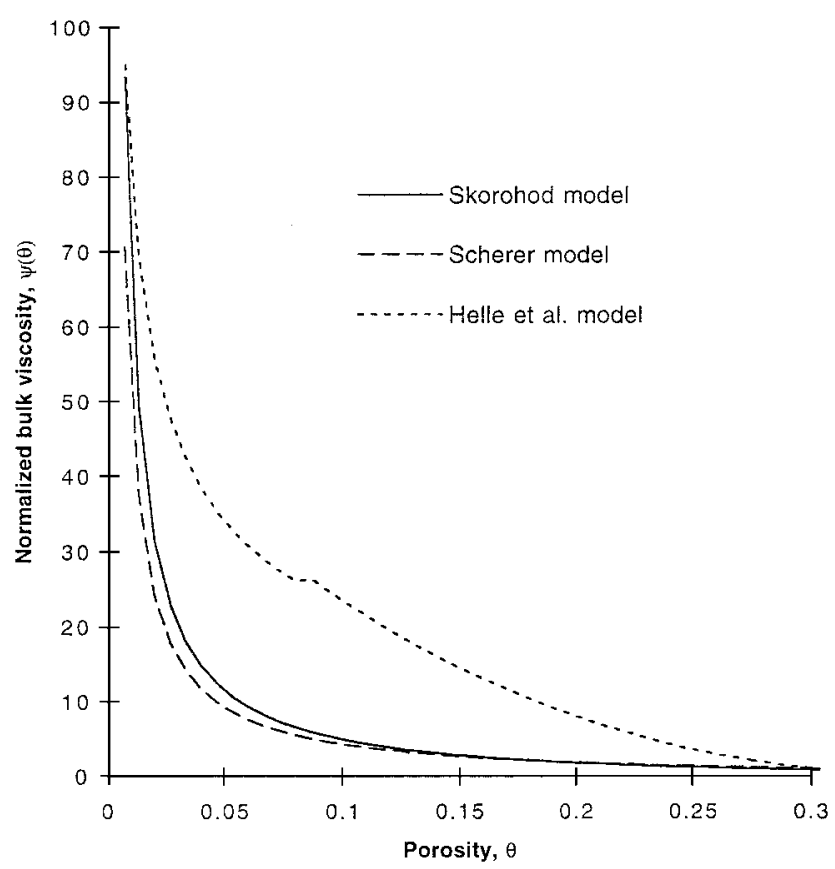

Fig. 1. Porosity dependence of the normalized bulk viscosity modulus $\psi(\theta)$.

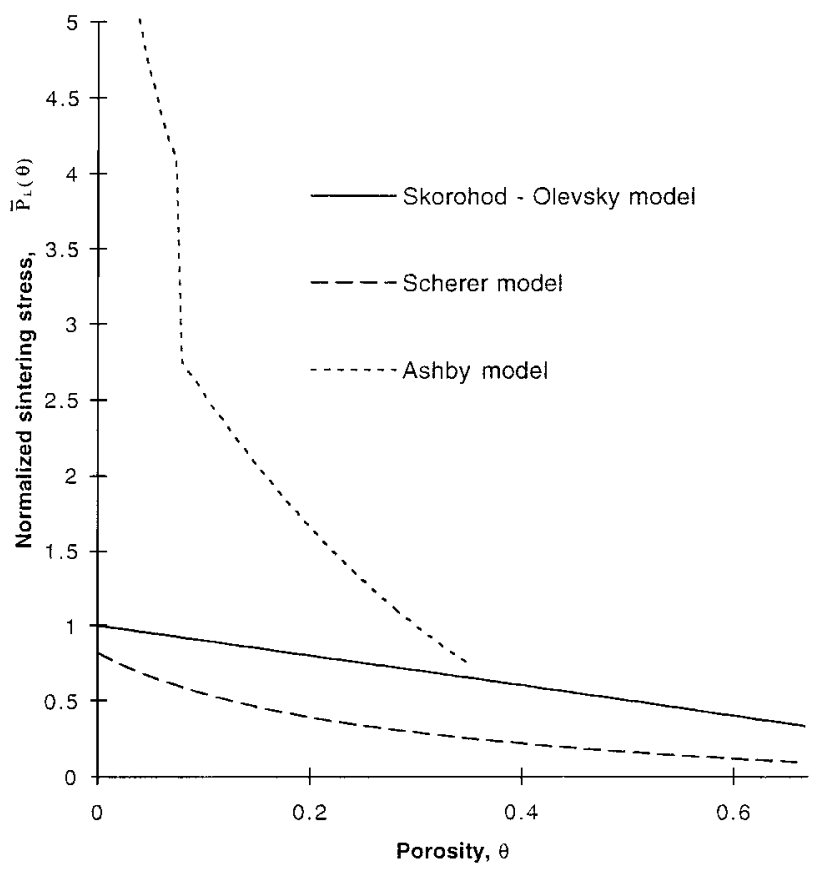

Fig. 2. Porosity dependence of the normalized effective sintering stress $\bar{P}_{\mathrm{L}}(\theta)$. 


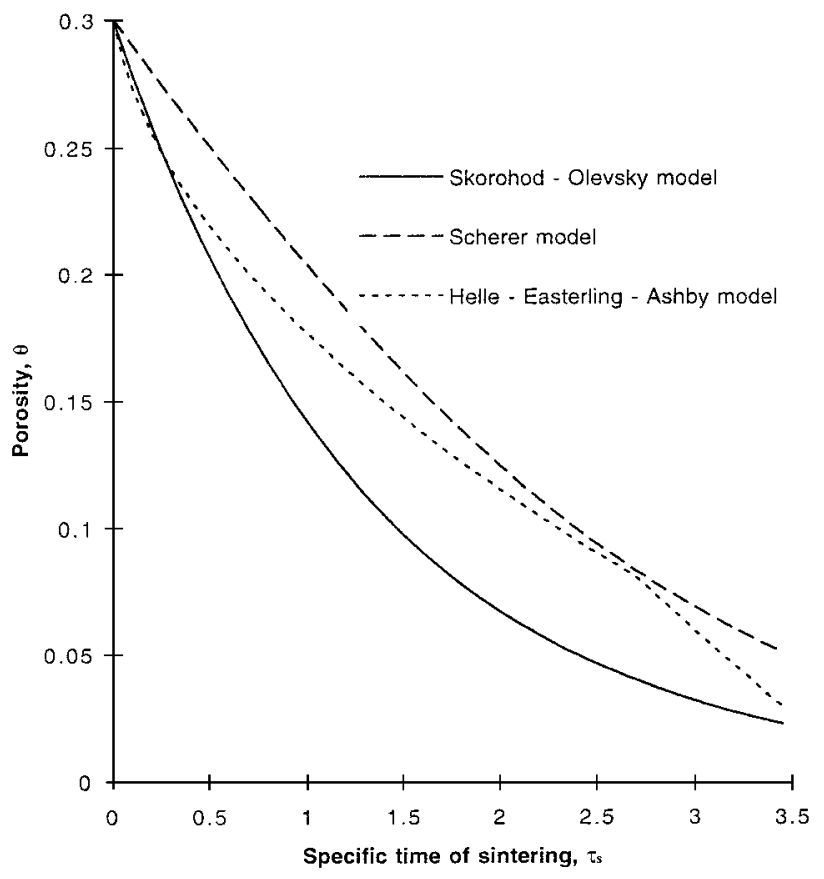

Fig. 3. Porosity evolution during free sintering vs. the specific time of sintering.

The limiting case of a skeleton with a rigid perfectly plastic behavior is also of interest. Such behavior can be obtained from the nonlinear-viscous law (30), with the strain rate sensitivity $m$ tending to zero. Combining (30) and (47) we obtain:

$$
e=-\frac{P_{\mathrm{L}}}{A} \frac{1}{\psi} W^{1-m}
$$

Therefore, when $m->0$, the relationship (52) can be written with consideration of (48), as:

$$
\frac{\tau_{0}}{P_{\mathrm{L} 0}}=\frac{\bar{P}_{\mathrm{L}}(\theta)}{\sqrt{2 \psi(\theta) \cdot(1-\theta)}}
$$

We have used the fact that, for $m=0, A=\sqrt{2} \tau_{o}$ in Eq. (30), where $\tau_{0}$ is the yield stress of the skeleton in shear.

For the three models of sintering discussed here, it turns out that there is a single value $\theta_{c}$ of the porosity for which Eq. (53) is satisfied (see Fig. 4). We shall call $\theta_{c}$ the critical porosity. The stability analysis represented below will show that for an initial porosity, such that $\theta_{o}>\theta_{c}$, the shrinkage rate is infinite. The porosity $\theta_{c}$ is obtained instantaneously (the inertia forces are neglected), and the sintering process stops afterwards. If the initial porosity is smaller than $\theta_{c}$, shrinkage does not occur. 


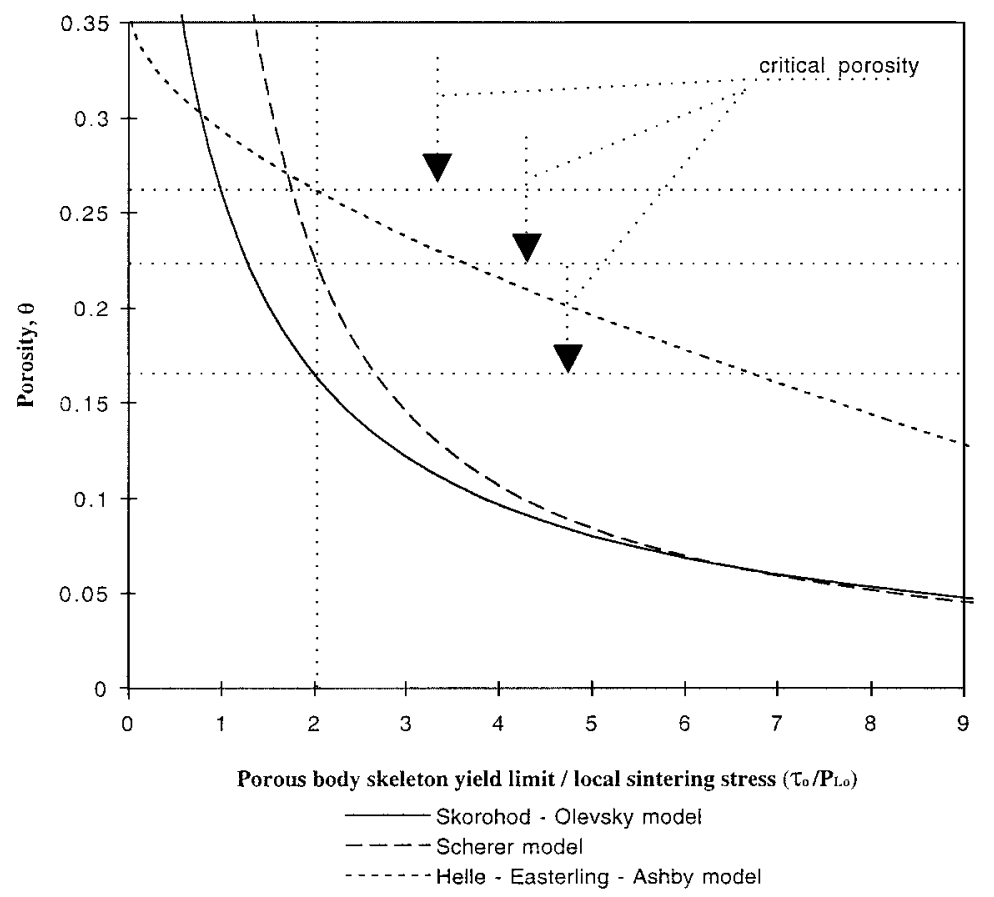

Fig. 4. Critical porosity vs. the ratio between the local sintering stress and the porous body skeleton's yield limit $P_{\mathrm{L} o} / \tau_{o}$. Rigid-plastic behavior is assumed for the skeleton.

The question of the role of plastic deformation as a primary sintering mechanism is a subject of a long-going discussion (see Brett and Seigle, 1963; Morgan, 1973, Schatt et al., 1986; German, 1996). For that reason, the analysis of sintering of a perfectly rigid-plastic material can be considered as a contribution to the abovementioned polemic, showing that plastic mechanism is highly unstable under sintering, because the stabilizing effects of the rate sensitivity are absent.

The fundamental uniform solution discussed above has a physical existence only if it can be proved that it is stable. If on the contrary this solution appears to be unstable, that means that a heterogeneity of the density will develop during the shrinkage process. In order to evaluate such possibilities, we shall analyze the stability of the fundamental solution using a perturbation method.

For the sake of simplicity, the stability analysis is developed for a particular geometry of the porous material. We shall consider a cylindrical specimen with an initially uniformly distributed density and with a radius small with respect to the length. The initial cross-section is uniform. A non-zero macroscopic compaction pressure may be applied. Inertia forces are neglected. Let us denote by $\sigma_{i j}^{(o)}(t)=$ $p(t) \delta_{i j}, \theta^{(o)}(t)$, and $\mathrm{e}^{(o)}(t)$ the stress, the porosity and the shrinkage rate corresponding to the fundamental solution associated with a uniform distribution of the initial density (porosity). $\theta^{(o)}(t)$ and $\mathrm{e}^{(o)}(t)$ are solutions of (39) and (47). We shall consider only the possibility of development of a heterogeneity in the direction $(x)$ of the 
cylindrical specimen's axis. A perturbed solution is considered in the form ( $i$ is the imaginary unit):

$$
Y(x, t)=Y^{(0)}(t)+\delta Y^{(0)} \exp (\lambda t) \exp (i \xi x)
$$

where $Y$ represents the stress, the porosity or the shrinkage rate. $\delta Y^{(0)}$ characterizes the amplitude of the perturbation, $\xi$ is the wave number. In general, $\bar{\lambda}$ is a complex number which can be time dependent. If $1 \exp (\bar{\lambda} t) 1$ decays with time, the problem is said to be linearly stable. This is equivalent to $\operatorname{Re}(\lambda)<0$, where $\chi=\bar{\lambda} t+\bar{\lambda}$.

The one-dimensional solution derived here is obtained with use of the same approximations that for the 1-D long wavelength solution of the simple tension problem of a thin rod with nonuniform cross-section (in particular, it is reminded that the compatibility equations for the strain are not rigorously satisfied because three dimensional effects are not exactly accounted for).

The perturbed solution of the sintering problem has the following form: the stress is hydrostatic and uniform:

$$
\sigma_{i j}(t)=p(t) \delta_{i j}
$$

$\theta(x, t)$ and $e(x, t)$ are solutions of the set of equations (39) and (47). Note that there is no spatial coupling in these equations.

After linearization of these equations, it is shown that

$$
\begin{aligned}
& \delta p=\frac{\mathrm{d}}{\mathrm{d} W}\left[\frac{\sigma(W)}{W}\right]^{(o)} \psi^{(o)} \mathrm{e}^{(o)} \delta W+\frac{\sigma\left(W^{(o)}\right)}{W^{(o)}}\left[\mathrm{e}^{(o)} \delta \psi+\psi^{(o)} \delta e\right]+\delta P_{\mathrm{L}}=0 \\
& \delta e=\frac{1}{\left(1-\theta^{(o)}\right)^{2}}\left[\lambda\left(1-\theta^{(o)}\right)+\dot{\theta}^{(o)}\right] \delta \theta
\end{aligned}
$$

It is reminded that the subscript $(o)$ refers to the fundamental homogeneous solution. We have set $\delta p=0$ because the pressure $p(t)$ is the one imposed at the boundaries of the specimen. It follows from the definition (16) of $W$ that:

$$
\delta W=\frac{\gamma^{(o)^{2}} \delta \varphi+2 \varphi(o) \gamma^{(o)} \delta \gamma+\mathrm{e}^{(o)} \delta \varphi+2 \varphi^{(o)} \mathrm{e}^{(o)} \delta e}{2\left(1-\theta^{(o)}\right) W^{(o)}}+\frac{W^{(o)}}{2\left(1-\theta^{(o)}\right)} \delta \theta
$$

This expression can be simplified because of $\gamma^{(o)}=0$ for the fimdamental solution. Finally, substituting (57), (58) into (56) and considering that and $\delta \psi=\psi_{\theta}^{\prime} \delta \theta, \psi_{\theta}^{\prime}=\frac{\mathrm{d} \psi}{\mathrm{d} \theta}$ and $P_{\mathrm{L} \theta}^{\prime}=\frac{\mathrm{d} P_{\mathrm{L}}}{\mathrm{d} \theta}$, we get:

$$
\Lambda \delta \theta=0
$$

with 


$$
\begin{aligned}
\Lambda= & \frac{\mathrm{d}}{\mathrm{d} W}\left[\frac{\sigma(W)}{W}\right]\left[\frac{\mathrm{e}^{2} \psi_{\theta}^{\prime}}{2(1-\theta) W}+\frac{2 \psi e[\lambda(1-\theta)+\dot{\theta}]}{2(1-\theta)^{3} W}+\frac{W}{2(1-\theta)}\right] \psi e \\
& +\frac{\sigma(W)}{W} \psi_{\theta}^{\prime} e+\frac{\sigma(W)}{W} \frac{\psi}{(1-\theta)^{2}}[\lambda(1-\theta)+\dot{\theta}]+P_{\mathrm{L} \theta}^{\prime}
\end{aligned}
$$

In order to obtain a non-trivial solution $\left(\delta_{\theta} \neq 0\right)$, the condition is:

$$
\Lambda=0
$$

which can be rewritten (taking into account that, for the fundamental solution, $\left.\frac{W}{\sigma(W) \psi e}=-\frac{1}{P_{\mathrm{L}}}\right)$ as:

$$
\frac{\lambda}{e}=\frac{2(1-\theta)\left[\frac{\bar{P}_{\mathrm{L}} \theta}{\bar{P}_{\mathrm{L}}}-\frac{\psi_{\theta}^{\prime}}{\psi}-\frac{1}{1-\theta}\right]-\kappa\left(\frac{\psi_{\theta}^{\prime}}{\psi}(1-\theta)+3\right)}{2(\kappa+1)}
$$

where $\kappa=\frac{\mathrm{d} \sigma / \mathrm{d} W}{\sigma(W)} W-1, \bar{P}_{\mathrm{L}}=\frac{P_{\mathrm{L}}}{P_{\mathrm{L} 0}}$ normalized sintering stress.

For material which obeys the power-law relationship (30) (which can be accepted as a generalization of both plastic and viscous flow mechanisms of deformation), $\kappa=m-1$, with the strain rate sensitivity $(m)$ satisfying the condition $0 \leqslant m \leqslant 1$. Then Eq. (62) can be written as:

$$
\frac{\lambda}{e}=\frac{2(1-\theta) \frac{\bar{P}_{\mathrm{L} \theta}^{\prime}}{\bar{P}_{\mathrm{L}}}-(1-\theta)(1+m) \frac{\psi_{\theta}^{\prime}}{\psi}-3 m+1}{2 m}
$$

For a negative value of $\lambda$ the perturbation mode is stable. As the shrinkage rate $(e)$ is negative under sintering, the process is stable if the right-hand side of (63) is positive. Using Eq. (63), the stability criterion can be written in the form:

$$
\frac{\mathrm{d}}{\mathrm{d} \theta}\left[\frac{\bar{P}_{\mathrm{L}}^{2}}{\psi^{1+m}(1-\theta)^{1-3 m}}\right]>0
$$

For the linear-viscous material properties, we have $m=1$ and the criterion (64) can be represented as follows:

$$
(1-\theta)\left[\frac{\bar{P}_{\mathrm{L} \theta}^{\prime}}{\bar{P}_{\mathrm{L}}}-\frac{\psi_{\theta}^{\prime}}{\psi}\right]>1
$$


For a perfect plastic material, $m=0$ and the criterion (63) indicates the possibility of two extreme modes $\lambda=+\infty$ or $\lambda=-\infty$ depending on the current porosity value $\theta>\theta_{c}$ and $\theta<\theta_{c}$, respectively, where $\theta_{c}$, is the critical porosity derived above.

Here, the results of the stability analysis are represented for three models of sintering: Scherer model (Scherer, 1977, 1979; Bordia and Scherer, 1988) (which is applied, in many cases, for the description of sintering of amorphous materials), Helle-Easterling-Ashby model (Helle et al., 1985; Ashby, 1990) (for these two models the expressions for $\bar{P}_{\mathrm{L}}$ and $\psi$ are given in Table 1) and Skorohod-Olevsky model with $\psi$ and $\bar{P}_{\mathrm{L}}$ given by Eqs. (A8) and (A31) of the Appendix.

For different values of the strain rate sensitivity, the left-hand part of inequality (64) is plotted in Figs. 5-7 for the above models. Fig. 5 indicates the stability of isothermal sintering for the Skorohod-Olevsky model. The Scherer model (Fig. 6) allows a weak instability of sintering for porosities $\theta>40 \%$ and linear-viscous properties of the porous body skeleton (this is a rare case of sintering of loose powder or highly-porous material). The Scherer model can be applied only for porosities $\theta>0.058$ which is omitted in the following discussion.

The break at $8 \%$ of porosity (Fig. 7) for Helle-Easterling-Ashby model is connected with a two-stage character of this model. The Helle-Easterling-Ashby model predicts an instability of sintering in the range of smaller porosities $(<8 \%)$. The instability is strong for higher nonlinearity of material properties.

\section{Skorohod - Olevsky model}

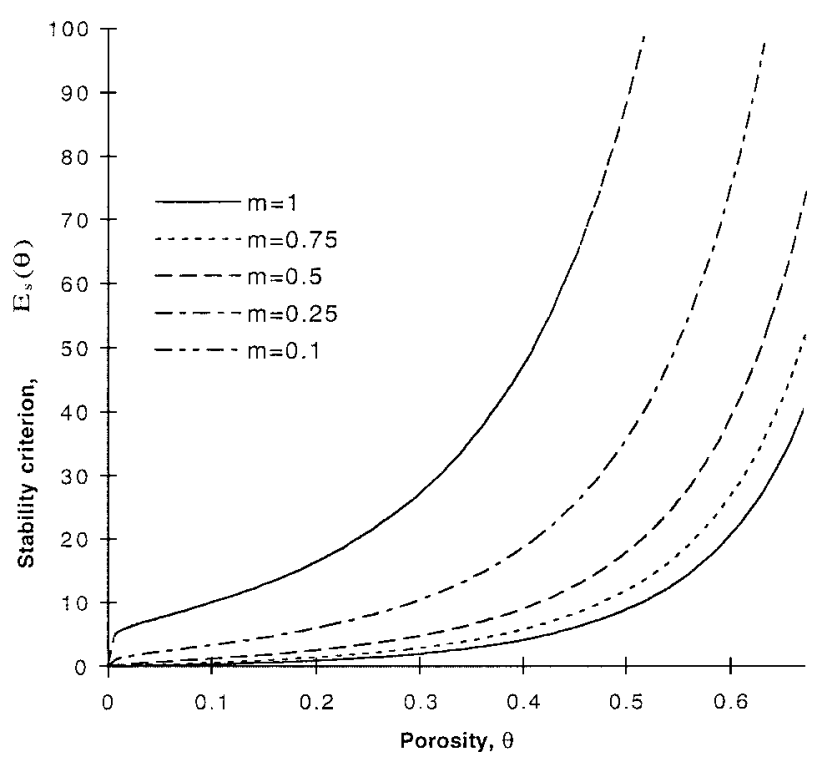

Fig. 5. Porosity dependence of the expression $E_{S}(\theta)=\frac{\mathrm{d}}{\mathrm{d} \theta}\left[\frac{\bar{P}_{\mathrm{L}}^{2}}{\psi^{1+m}(1-\theta)^{1-3 m}}\right]$ appearing in the stability criterion (64) $\left(E_{s}(\theta)>0\right)$ obtained for the Skorohod-Olevsky model. 


\section{Scherer model}

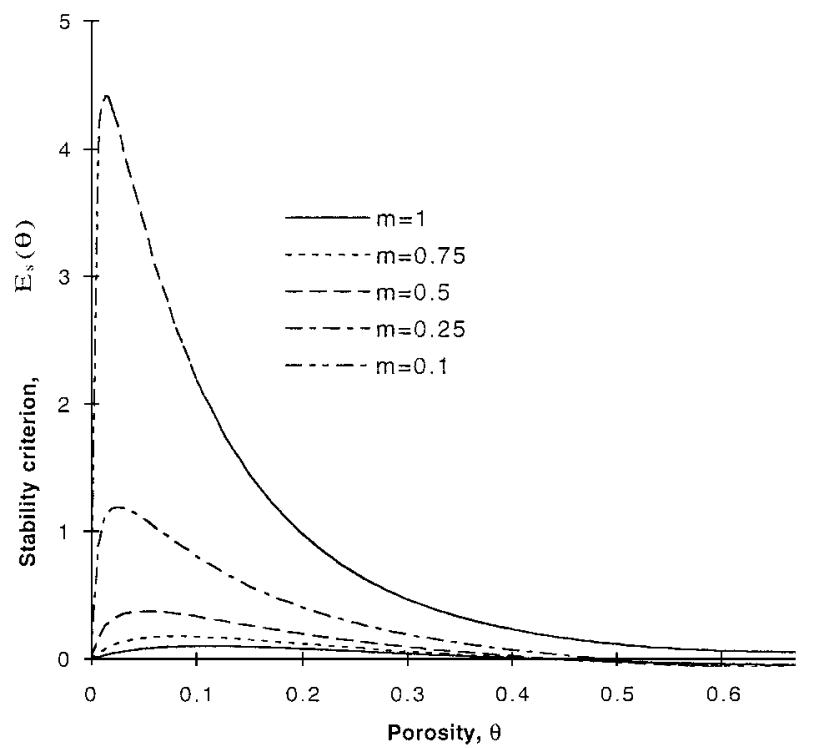

Fig. 6. Porosity dependence of the expression $E_{S}(\theta)=\frac{\mathrm{d}}{\mathrm{d} \theta}\left[\frac{\bar{P}_{\mathrm{L}}^{2}}{\psi^{1+m}(1-\theta)^{1-3 m}}\right]$ appearing in the stability criterion (64) $\left(E_{S}(\theta)>0\right)$ obtained for the Scherer model.

For linear-viscous material properties $(m=1)$, the results of the stability analysis for all the three models are shown in Fig. 8. A combined graph of the porosity and the normalized perturbation growth rate evolution vs. the specific time of sintering is represented in Fig. 9.

Reliable models should provide stable solutions for the case of isothermal sintering (when no physically destabilizing effects are present). Thus, besides a pure experimental verification of the predicted shrinkage kinetics (Fig. 3), the stability analysis results given in Fig. 8 can serve as a "weakness indicator" for the considered models of sintering. Accordingly, the Scherer model should be considered as less physically reliable for higher porosities of about $40 \%$, both Skorohod-Olevsky and Scherer models are less reliable for porosities approaching zero, and the HelleEasterling-Ashby model's "point of weakness" is $\theta=0.08$ corresponding to the singularity discussed above.

\subsection{Stability analysis accounting for thermomechanical coupling}

It follows from (37) and (44) (assuming that $\beta=1$ and taking into consideration that $\gamma=0$ in free sintering):

$$
\rho c \dot{T}=-P_{\mathrm{L}} e
$$


Helle - Easterling - Ashby model

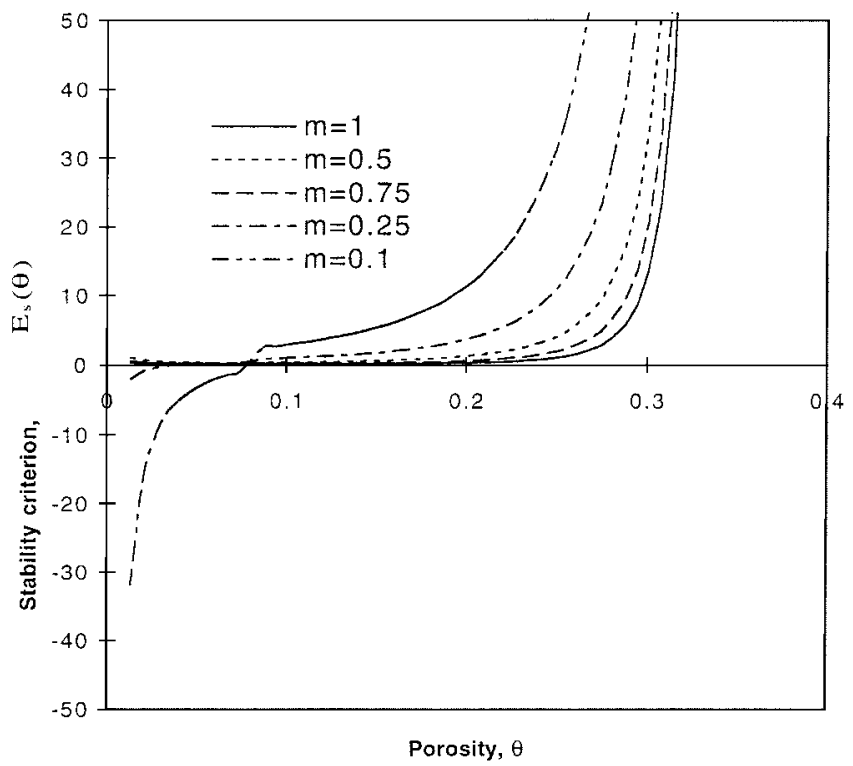

Fig. 7. Porosity dependence of the expression $E_{S}(\theta)=\frac{\mathrm{d}}{\mathrm{d} \theta}\left[\frac{\bar{P}_{\mathrm{L}}^{2}}{\psi^{1+m}(1-\theta)^{1-3 m}}\right]$ appearing in the stability criterion (64) $\left(E_{s}(\theta)>0\right)$ obtained for linear-viscous material. The dependence corresponding to the Helle-Easterling-Ashby model has a singularity at $\theta=0.08$.

where $\dot{T}$ is the time derivative of the temperature; $c$ and $\rho$ are the effective heat capacity and density given by (42) and (43), respectively.

Substituting Eqs. (39), (42) and (43) into Eq. (66), one obtains:

$$
\rho_{0} c_{0} \bar{c} \dot{T}=-P_{\mathrm{L}} \frac{\dot{\theta}}{1-\theta}
$$

Simultaneously, from Eqs. (52) and (39) we have:

$$
\dot{\theta}=-\left(\frac{P_{\mathrm{L}}}{A}\right)^{\frac{1}{m}} \frac{(1-\theta)^{\frac{3 m-1}{2 m}}}{\psi^{\frac{m+1}{2 m}}}
$$

Perturbing Eqs. (67) and (68) with respect to porosity and temperature, taking into consideration that the effective sintering stress depends both on temperature and porosity as follows:

$$
P_{\mathrm{L}}=P_{\mathrm{L} 0}(T) \bar{P}_{\mathrm{L}}(\theta)
$$

one obtains a set of equations: 


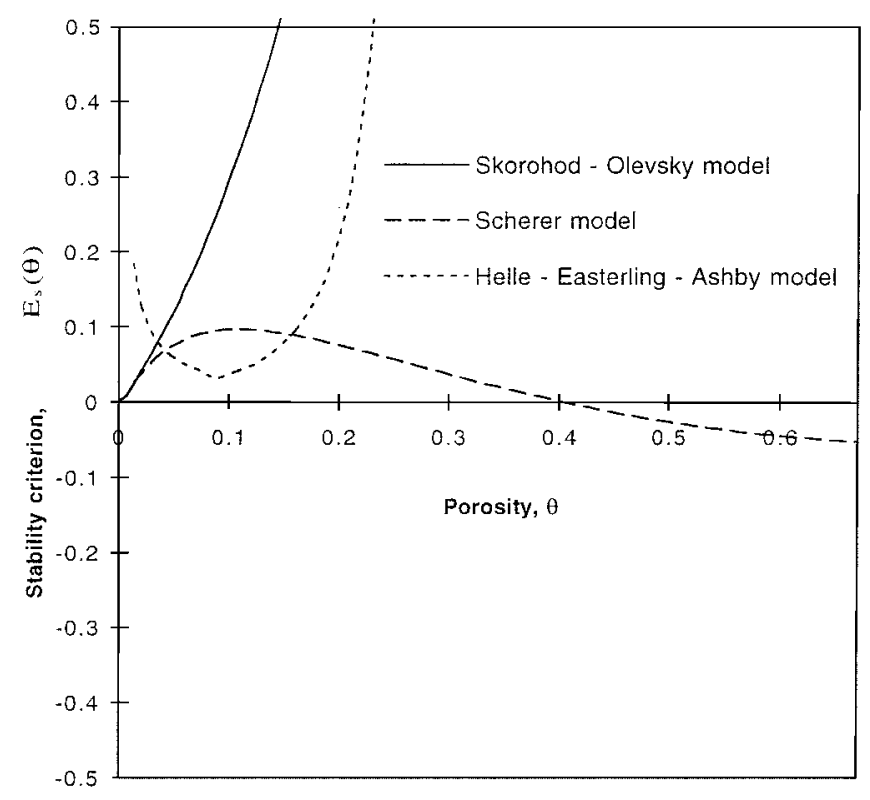

Fig. 8. Porosity dependence of the expression $E_{S}(\theta)=\frac{\mathrm{d}}{\mathrm{d} \theta}\left[\frac{\bar{P}_{\mathrm{L}}^{2}}{\psi^{1+m}(1-\theta)^{1-3 m}}\right]$ appearing in the stability criterion (64) $\left(E_{S}(\theta)>0\right)$ obtained for linear-viscous material. The dependence corresponding to the Helle-Easterling-Ashby model has a singularity at $\theta=0.08$.

$$
\left\{\begin{array}{l}
{\left[\frac{c_{0 \mathrm{~T}}^{\prime}}{c_{0}}+\frac{\rho_{0 \mathrm{~T}}^{\prime}}{\rho_{0}}-\frac{P_{\mathrm{L} 0 \mathrm{~T}}^{\prime}}{P_{\mathrm{L} 0}}-\frac{(1-\theta)^{2} \rho_{0} c}{P_{\mathrm{L}}} \frac{\lambda}{\dot{\theta}}\right] \delta T^{(o)}+\left[\frac{\bar{c}_{\theta}^{\prime}}{\bar{c}}-\frac{\bar{P}_{\mathrm{L} \theta}^{\prime}}{\bar{P}_{\mathrm{L}}}-\frac{2}{1-\theta} \frac{\lambda}{\dot{\theta}}\right] \delta \theta^{(o)}=0} \\
2\left[\frac{P_{\mathrm{L} 0 \mathrm{~T}}^{\prime}}{P_{\mathrm{L} 0}}-\frac{A_{\mathrm{T}}^{\prime}}{A}\right] \delta T^{(o)}+\left[2 \frac{\bar{P}_{\mathrm{L} \theta}^{\prime}}{\bar{P}_{\mathrm{L}}}+\frac{1-3 m}{1-\theta}-(m+1) \frac{\psi_{\theta}^{\prime}}{\psi}-\frac{2 m \lambda}{\dot{\theta}}\right] \delta \theta^{(o)}=0
\end{array}\right.
$$

where:

$$
\begin{aligned}
c_{0 \mathrm{~T}}^{\prime} & =\frac{\mathrm{d} c_{0}\left(T^{(o)}\right)}{\mathrm{d} T}, \rho_{0 \mathrm{~T}}^{\prime}=\frac{\mathrm{d} \rho_{0}\left(T^{(o)}\right)}{\mathrm{d} T}, P_{\mathrm{L} 0 \mathrm{~T}}^{\prime}=\frac{\mathrm{d} P_{\mathrm{L} 0}\left(T^{(o)}\right)}{\mathrm{d} T}, \bar{c}_{\theta}^{\prime}=\frac{\mathrm{d} \bar{c}\left(\theta^{(o)}\right)}{\mathrm{d} \theta}, \bar{P}_{\mathrm{L} \theta}^{\prime} \\
& =\frac{\mathrm{d} \bar{P}_{\mathrm{L}}\left(\theta^{(o)}\right)}{\mathrm{d} \theta}, A_{\mathrm{T}}^{\prime}=\frac{\mathrm{d} A\left(T^{(o)}\right)}{\mathrm{d}}
\end{aligned}
$$

As before, the subscript ${ }^{(o)}$ refers to the fundamental homogeneous solution. To have a non-trivial solution $\left(\delta T^{(o)}, \delta \theta^{(o)}\right) \neq(0,0)$ of the linear equations $(70)$, the determinant is set equal to zero. A quadratic equation in terms of $\lambda / e$ is obtained:

$$
B_{1}\left(\frac{\lambda}{e}\right)^{2}+B_{2}\left(\frac{\lambda}{e}\right)+B_{3}=0
$$




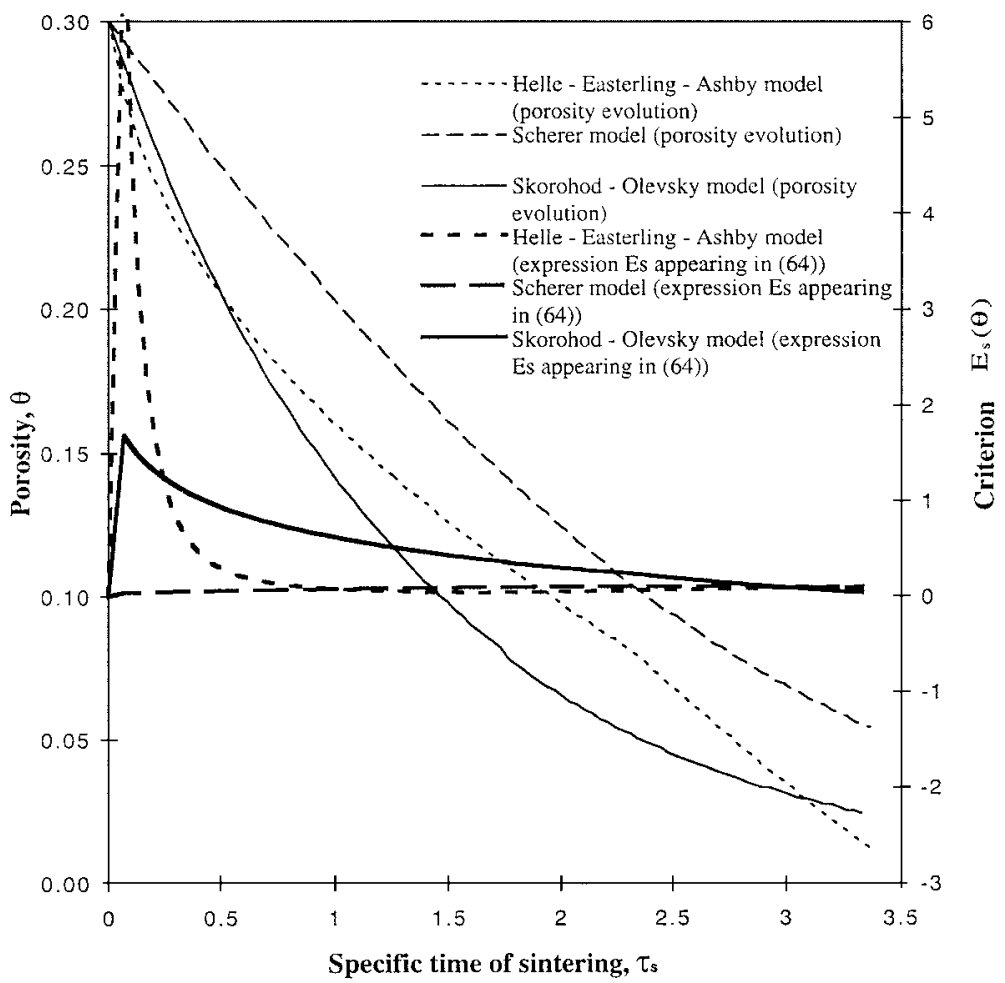

Fig. 9. Evolution of porosity $\theta$ and specific time dependence of the expression $E_{S}(\theta)=\frac{\mathrm{d}}{\mathrm{d} \theta}\left[\frac{\bar{P}_{\mathrm{L}}^{2}}{\psi^{1+m}(1-\theta)^{1-3 m}}\right]$ appearing in the stability criterion $(64)\left(E_{s}(\theta)>0\right)$ obtained for linear-viscous material. The dependence corresponding to the Helle-Easterling-Ashby model has a singularity at $\theta=0.08$.

where

$$
\left\{\begin{aligned}
B_{1}= & -2 m \frac{\rho_{0} c}{P_{\mathrm{L}}} \\
B_{2}= & \frac{2}{1-\theta}\left(\frac{A_{\mathrm{T}}^{\prime}}{A}-\frac{P_{\mathrm{L} 0 \mathrm{~T}}^{\prime}}{P_{\mathrm{L} 0}}\right)+\frac{(1-\theta) \rho_{0} c}{P_{\mathrm{L}}}\left(2 \frac{\bar{P}_{\mathrm{L} \theta}^{\prime}}{\bar{P}_{\mathrm{L}}} \frac{1-3 m}{1-\theta}-(m+1) \frac{\psi_{\theta}^{\prime}}{\psi}\right) \\
+ & \frac{2 m}{1-\theta}\left(\frac{c_{0 \mathrm{~T}}^{\prime}}{c_{0}}+\frac{\rho_{0 \mathrm{~T}}^{\prime}}{\rho_{0}}-\frac{P_{\mathrm{L} 0 \mathrm{~T}}^{\prime}}{P_{\mathrm{L} 0}}\right) \\
B_{3}= & -2\left(\frac{A_{\mathrm{T}}^{\prime}}{A}-\frac{P_{\mathrm{L} 0 \mathrm{~T}}^{\prime}}{P_{\mathrm{L} 0}}\right)\left(\frac{\bar{c}_{\theta}^{\prime}}{\bar{c}}-\frac{\bar{P}_{\mathrm{L} \theta}^{\prime}}{\bar{P}_{\mathrm{L}}}-\frac{2}{1-\theta}\right)-\left(\frac{c_{0 \mathrm{~T}}^{\prime}}{c_{0}}+\frac{\rho_{0 \mathrm{~T}}^{\prime}}{\rho_{0}}-\frac{P_{\mathrm{L} 0 \mathrm{~T}}^{\prime}}{P_{\mathrm{L} 0}}\right) \\
& \left(2 \frac{\bar{P}_{\mathrm{L} \theta}}{\bar{P}_{\mathrm{L}}}+\frac{1-3 m}{1-\theta}-(m+1) \frac{\psi_{\theta}^{\prime}}{\psi}\right)
\end{aligned}\right.
$$

If, for simplicity, $P_{\mathrm{L} 0 \mathrm{~T}}^{\prime}=0, \rho_{0 \mathrm{~T}}^{\prime}=0$ and $c_{0 \mathrm{~T}}^{\prime}=0$, then: 


$$
\left\{\begin{array}{l}
B_{1}=-2 m \frac{\rho_{0} c}{P_{\mathrm{L}}} \\
B_{2}=\frac{2}{1-\theta} \frac{A_{\mathrm{T}}^{\prime}}{A}+\frac{(1-\theta) \rho_{0} c}{P_{\mathrm{L}}}\left(2 \frac{\bar{P}_{\mathrm{L} \theta}^{\prime}}{\bar{P}_{\mathrm{L}}}+\frac{1-3 m}{1-\theta}-(m+1) \frac{\psi_{\theta}^{\prime}}{\psi}\right) \\
B_{3}=-2 \frac{A_{\mathrm{T}}^{\prime}}{A}\left(\frac{\bar{c}_{\theta}^{\prime}}{\bar{c}}-\frac{\bar{P}_{\mathrm{L} \theta}^{\prime}}{\bar{P}_{\mathrm{L}}}-\frac{2}{1-\theta}\right)
\end{array}\right.
$$

Note that $B_{1}<0$. If $B_{3}>0$, Eq. (71) has two real roots, one positive and one negative. If $B_{3}<0$, the real parts of both roots have the sign of $B_{2}$. Thus the process is not stable if $B_{3}<0, B_{2}<0$, and is stable if $B_{3}<0, B_{2}>0$. It is always unstable when $B_{3}>0$.

Here, following Ashby (1990), the pre-exponential factor in the creep power-law is given by:

$$
A=A_{0} \exp \left(\frac{A_{1}}{T}+A_{2}\right)
$$

where:

$$
A_{1}=\frac{Q_{c}}{R_{g}}, A_{2}=-\frac{2 Q_{c}}{R_{g} T_{m}}
$$

$\sigma_{\text {ref }}$ is a reference stress, $Q_{c}$ is an activation energy, $R_{g}$ is the gas constant, $T_{m}$ is the melting temperature.

Hence:

$$
\frac{A_{\mathrm{T}}^{\prime}}{A}=-\frac{Q_{c}}{R_{g} T^{2}}<0
$$

Thus, a sufficient condition of stability can be formulated as follows:

$$
\left\{\begin{array}{l}
\frac{(1-\theta)^{2} \bar{c}}{\bar{P}_{\mathrm{L}}} \frac{\mathrm{d}}{\mathrm{d} \theta}\left\{\ln \left[\frac{\bar{P}_{\mathrm{L}}^{2}}{\psi^{1+m}(1-\theta)^{1-3 m}}\right]\right\}>\frac{2 Q_{c} P_{\mathrm{L} 0}}{\rho_{0} R_{g} T^{2} c_{0}} \quad\left(B_{2}>0\right) \\
G(\theta)=\frac{\mathrm{d}}{\mathrm{d} \theta}\left[\frac{\bar{c}}{\bar{P}_{\mathrm{L}}}(1-\theta)^{2}\right]<0 \quad\left(B_{3}<0\right)
\end{array}\right.
$$

In the case, when $A_{\mathrm{T}}^{\prime}=0$ (we have no temperature dependence of all the material properties), the first inequality in (77) is reduced to (64).

Fig. 10 shows that the sufficient condition of stability (77) is valid only for the Skorohod-Olevsky model, as the function $G(\theta)$ is always positive for Scherer and Helle-Easterling-Ashby models (here it is assumed that $\bar{c}=1-\theta$ ).

The process is always unstable when $B_{2}<0$. Therefore an instability criterion can be formulated as follows if $P_{\mathrm{L}}$ and $c$ are not dependent on $T$ : 


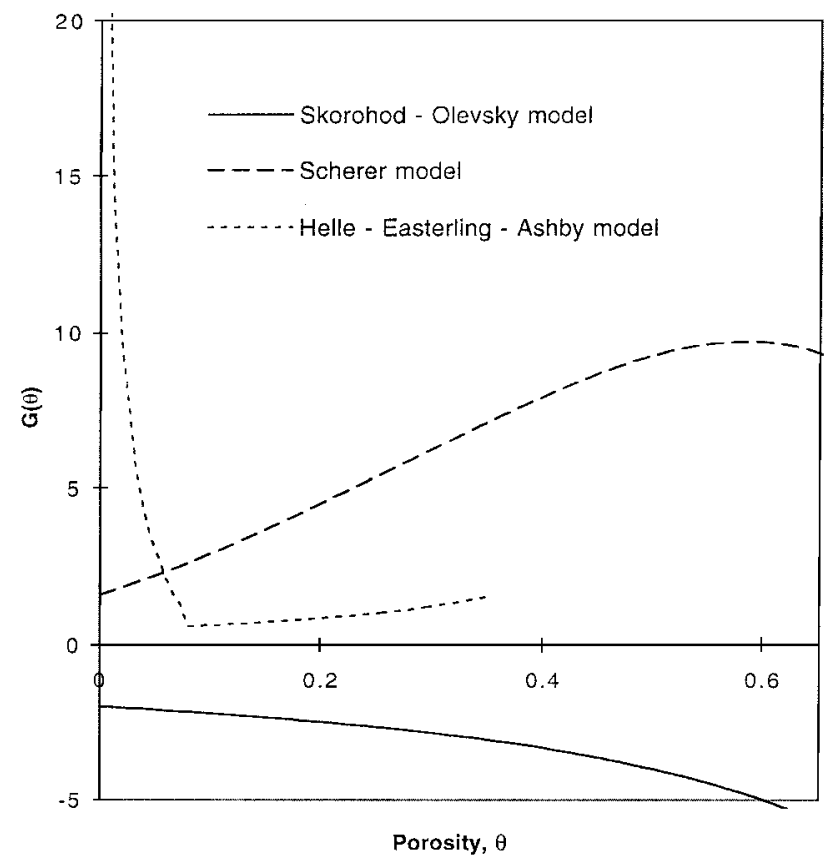

Fig. 10. Function $G(\theta)$ (Porosity dependence of the coefficient $B_{3}$ ).

$$
\frac{(1-\theta)^{2} \bar{c}}{\bar{P}_{\mathrm{L}}} \frac{\mathrm{d}}{\mathrm{d} \theta}\left\{\ln \left[\frac{\bar{P}_{\mathrm{L}}^{2}}{\psi^{1+m}(1-\theta)^{1-3 m}}\right]\right\}<\frac{2 Q_{c} P_{\mathrm{L} 0}}{\rho_{0} R_{g} T^{2} c_{0}}
$$

From condition $\left(77^{*}\right)$ it follows that instability is favored for materials with:
a. small heat capacity;
b. small theoretical density;
c. high activation energy of the power-law creep;
d. high surface tension;
e. small particle size;
f. low sintering temperature.

Item (f) indicates that sintering is more unstable at the early stages of densification which is a known phenomenon (Skorohod and Solonin, 1984; Bordia and Jagota, 1993).

It should be noted that the above-mentioned considerations are valid for materials with a negligible grain size evolution under sintering. However, this factor can cause a considerable instability of the process which is often connected with the Ostwald ripening effect (Chen and Fan, 1996). An incorporation of the grain growth kinetics into stability criterion is an object of further investigations. 
For amorphous (e.g. glass) material, the linear-viscosity formalism should be applied $(m=1)$, and the first inequality of (77) can be rewritten as follows:

$$
\frac{1-\theta}{\theta}>\frac{8 \alpha}{9 \rho_{0} c_{0} r_{0}}\left|\frac{A_{\mathrm{T}}^{\prime}}{A}\right|
$$

Here $r_{0}$ is the particle radius, $A$ corresponds to the shear viscosity modulus of the substance, $\alpha$ is the surface tension. Expressions (A8) and (A31) (see the Appendix) of the Skorohod-Olevsky model were used for the derivation of inequality (78). It is assumed that $\bar{c}=1-\theta$.

It can be shown that, for borosilicate glass, the stability criterion (78) is always satisfied for real processing conditions (for porosities $\theta<30 \%$ ). Using the following values for material parameters: $\alpha=0.15 \mathrm{~N} / \mathrm{m}, \rho_{0}=2130 \mathrm{~kg} / \mathrm{m}^{3}$ (Rahaman and DeJonghe, 1990), $c_{o}=1255 \mathrm{j} / \mathrm{kg}^{\circ} \mathrm{K}$ (Goldsmith et al., 1961), $r_{0}=10^{-5} \mathrm{~m}$, one can see that inequality (78) is satisfied for $\theta<30 \%$ if $\left|\frac{A_{T}^{\prime}}{A}\right|<400 \frac{1}{K}$. For borosilicate glass, $\mid \frac{A_{\mathrm{T}}^{\prime}}{A}=\frac{1.75 \times 10^{4}}{T^{2}} \mathrm{~K}$ (Scherer, 1977) which satisfies inequality (78) for the entire range of possible sintering temperatures. Values of $\left|\frac{A_{\mathrm{T}}^{\prime}}{A}\right|$ higher than $400 \frac{1}{K}$ can be achieved for rapidly crystallizing glasses when the viscosity quickly increases with the temperature due to the increase of the amount of a crystalline phase (Kingery, 1967; Panda et al., 1989).

For free sintering of crystalline bodies, diffusional mechanisms of material flow are dominant (German, 1996). For an analysis of the sintering stability for such materials, the bulk viscosity modulus and the rate sensitivity constant ( $m \sim 1$ in this case; see Cocks, 1994) should be associated with the corresponding diffusion parameters.

It should be noted that an introduction of the temperature-dependent exponent $m$ would enhance the model (which would correspond to the Norton-Hoff model of creep). This idea is considered as a subject of further investigations.

\subsection{Stability of isostatic pressing}

The stability criteria (64) and (77) and the instability criterion (77) can be applied for the stability analysis of isostatic pressing. In doing so, we have to set $P_{\mathrm{L}}=P_{\mathrm{L} 0}=P_{h}$, where $P_{h}$ is the externally applied hydrostatic pressure. The first expression in criterion (77) is modified as follows:

$$
(1-\theta)^{3}\left[\frac{1-3 m}{1-\theta}-\frac{\psi_{\theta}^{\prime}}{\psi}\right]>\frac{2 Q_{c} P_{h}}{(m+1) R_{g} \rho_{0} c_{0} T^{2}}
$$

For hot isostatic pressing of powders, it is appropriate to use the function $\psi(\theta)$ in the form suggested by Ponte Castaneda (1991):

$$
\psi=\frac{2}{3}\left(\frac{1-\theta^{m}}{m \theta^{m}}\right)^{\frac{2}{m+1}}
$$


In the case of linear-viscous rheology, the latter expression is reduced to an expression derived by Mackenzie (1950).

Substituting Eq. (80) into inequality (79), we obtain:

$$
(1-\theta)^{3}\left[\frac{m}{\theta\left(1-\theta^{m}\right)}+\frac{1-3 m}{2(1-\theta)}\right]>\frac{Q_{c} P_{h}}{R_{g} \rho_{0} c_{0} T^{2}}
$$

The stability criterion (81) is illustrated in Fig. 11. An instability is possible for the high values of the porosity. When $m$ is decreased (higher nonlinear response) instability is favored for $\theta<0.29$, while stabilizing effect are observed for $\theta>0.29$.

In Fig. 12 ("stability map"), the results of the stability analysis for nickel powder in accordance with criterion (81) are shown. Here the following material parameter values have been used (Ashby, 1990) (particle radius is $10^{-5} \mathrm{~m}$ ): $Q_{c}=284 \mathrm{~kJ} / \mathrm{mol}$, $\rho_{o}=8900 \mathrm{~kg} / \mathrm{m}^{3}, m=0.109, c_{o}=33 \mathrm{~J} /(\mathrm{mol} \mathrm{K})$ (Goldsmith et al., 1961). It is demonstrated how a minimum critical porosity value which corresponds to a possible instability can be determined. For example, for the external pressure of $60 \mathrm{MPa}$ and the temperature of $1000 \mathrm{~K}$, the stable mode of the HIPing is guaranteed only for a porosity smaller than $4 \%$.

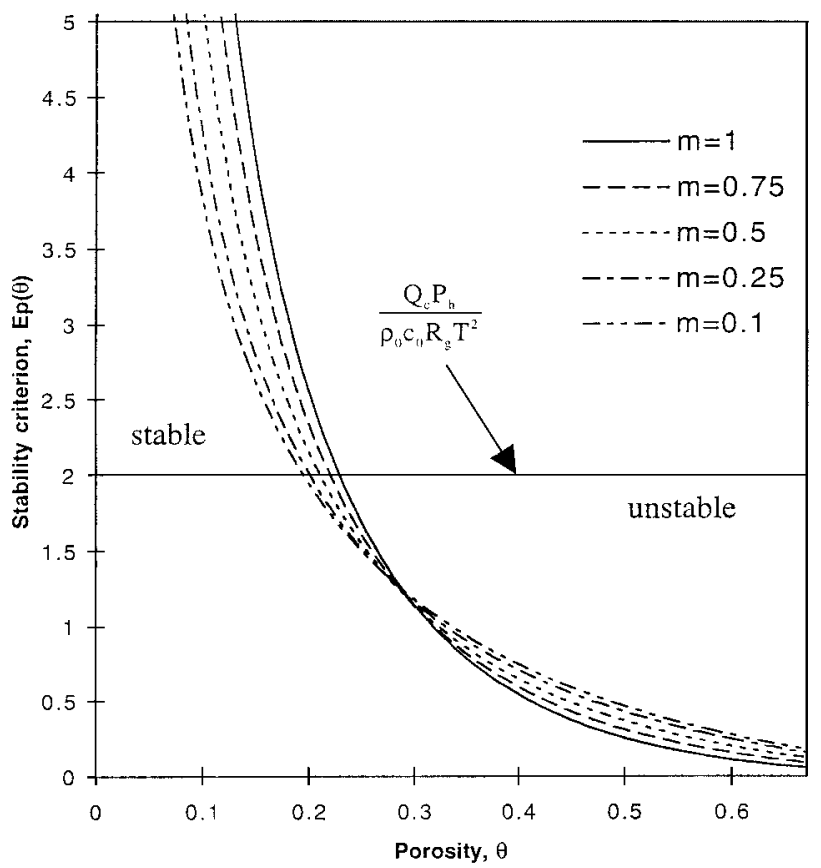

Fig. 11. Porosity dependence of the expression $E_{p}(\theta)=(1-\theta)^{3}\left[\frac{m}{\theta\left(1-\theta^{m}\right)}+\frac{1-3 m}{2(-\theta)}\right]$ appearing in the stability criterion Eq. (81) $\left(E_{p}(\theta)>\frac{Q_{Q} P_{h}}{\rho_{o} R_{o} R_{g} T^{2}}\right.$ for isostatic pressing. 


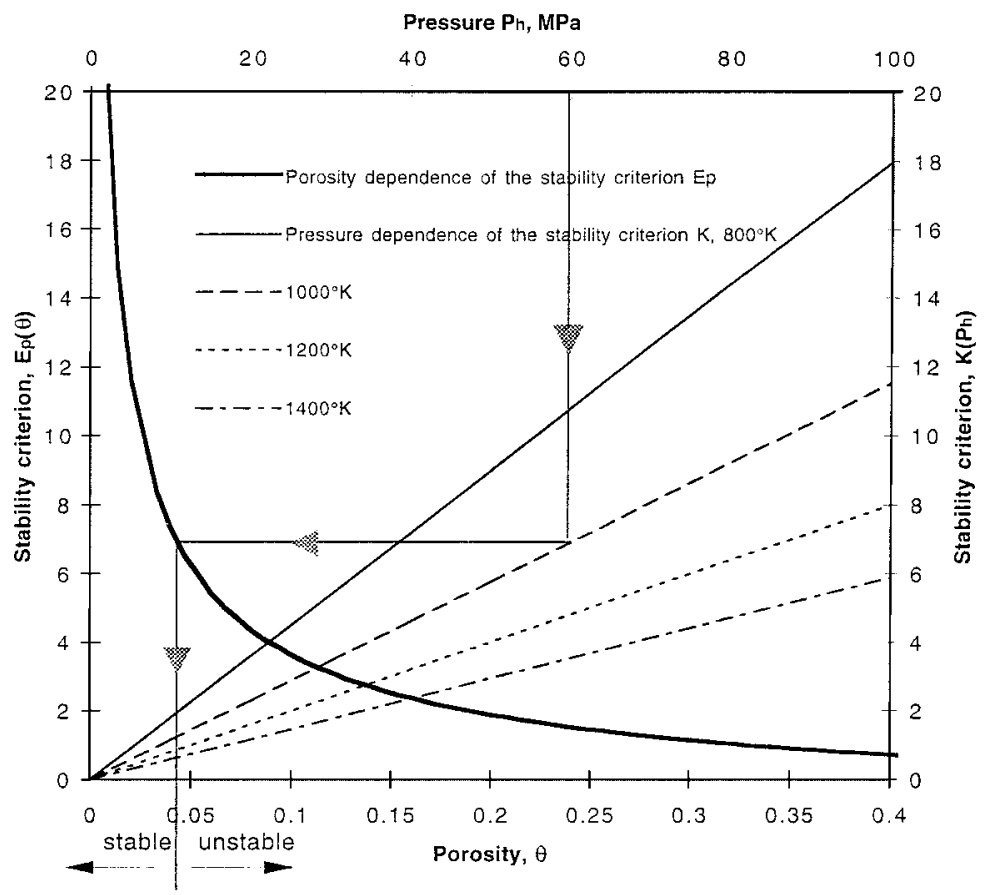

Fig. 12. Stability map for hot isostatic pressing of nickel powder. Particle radius is $10 \mu \mathrm{m}, Q_{c}=284 \mathrm{~kJ} /$ $\mathrm{mol}, m=0.109, c_{0}=33 \mathrm{~J} /(\mathrm{mol} \mathrm{K}), E_{p}(\theta)=(1-\theta)^{3}\left[\frac{m}{\theta\left(1-\theta^{m}\right)}+\frac{1-3 m}{2(-\theta)}\right]$. The curve corresponds to the expression $E_{p}(\theta)=(1-\theta)^{3}\left[\frac{m}{\theta\left(1-\theta^{m}\right)}+\frac{1-3 m}{2(-\theta)}\right]$ appearing in the stability criterion Eq. (81) $E_{p}(\theta)>\frac{Q_{c} P_{h}}{\rho_{o} c_{o} R_{g} T^{2}}$ for isostatic pressing. Linear lines correspond to the function $K\left(P_{h}\right)=\frac{Q_{c} P_{h}}{\rho_{o} c_{o} R_{g} T^{2}}$ for values of the temperature ranging from 800 to $1400 \mathrm{~K}$. An illustration is given for $P_{h}=60 \mathrm{MPa}$, and $T=1000 \mathrm{~K}$. It is seen that the process is stable for $\theta<0.04$ and unstable for $\theta>0.04$.

\section{Conclusions}

1. It is shown that isothermal sintering is always stable as described by Skorohod-Olevsky model if the self-heating due to the mechanical work is neglected. Scherer model predicts stability of isothermal sintering for porosities less than $40 \%$.

Helle-Easterling-Ashby model indicates unstable modes of sintering for porosities smaller than $8 \%$ and high nonlinearity of the constitutive properties.

2. A criterion of stability for sintering model accounting for thermomechanical coupling is derived. In the framework of this criterion, critical conditions of instability can be realized in terms of the temperature dependence of material parameters.

3. Possible instability of sintering is indicated for rapidly crystallizing glass powders. 
4. A criterion of stability of isostatic pressing is obtained. Possible instability is indicated for hot isostatic pressing of nickel powder.

\section{Acknowledgements}

The support of the NSF Institute for Mechanics and Materials, University of California, San Diego is gratefully acknowledged.

\section{Appendix: Porosity dependence of the constitutive parameters}

To obtain the overall effective response of a porous viscoplastic material in terms of the thermomechanical and geometrical characteristics of the constituents is in general a difficult task (see, for example, for linear-viscous materials, Mackenzie, 1950; Skorohod, 1972; Hsueh et al., 1986; Venkatachari and Raj, 1986; Ashby, 1990; Du and Cocks, 1992a,b; for power-law creep responses, and, Wilkinson and Ashby, 1975; Helle et al., 1985; Ponte Castaneda, 1991; Sofronis and McMeeking, 1992; Lee and Mear, 1992).

Examples of expressions for $\psi(\theta)$ obtained by different authors are represented in Table 1.

\section{A.1. Derivation of $\varphi(\theta)$ and $\psi(\theta)$}

We present here a micro-macro approach developed by Skorohod (1972) in the case of a linear-viscous material. Explicit expressions for $\varphi(\theta)$ and $\psi(\theta)$ are obtained once the effective shear and bulk moduli have been estimated.

\section{A.1.1. Determination of the effective shear modulus}

A first estimate of the effective shear modulus $\eta$ can be obtained by assuming that the pores are spherical voids embedded in a connected matrix. This type of closed porosity corresponds to the late stages of sintering where pores are not connected.

The material in the matrix is linear-viscous with viscosity $\eta_{0}$ and is incompressible. Using a differential scheme (see, for example, Skorohod, 1972) provides the following effective shear modulus:

$$
\eta=\eta_{0}(1-\theta)^{5 / 3}
$$

In the earlier stages of the sintering process, the porosity is open. Skorohod assumed then a cylindrical shape for the pores and found the following estimate of $\eta$ :

$$
\eta=\eta_{0}(1-\theta)^{2}
$$


Considering that the exponents $5 / 3$ and 2 in expressions (A1) and (A2) are close, Skorohod has assumed that the expression (A2) should be relevant for both early and late stages of sintering. Then the comparison of (A2) with Eq. (13) leads to:

$$
\varphi(\theta)=(1-\theta)^{2}
$$

\section{A.1.2. Determination of the effective bulk modulus}

The effective bulk modulus $\zeta$ is determined with use of a self-consistent scheme. For an applied macroscopic pressure $p$, we have:

$$
\dot{\varepsilon}_{i i}=p / \zeta
$$

The left-hand part of this expression is the trace of the macroscopic strain rate. Consider now an isolated pore and replace its surrounding by an infinite uniform medium with bulk and shear moduli $\zeta$ and $\eta$, respectively (this is the so-called equivalent homogeneous medium). The response of the isolated pore to a pressure $p$ applied at infinity is easily obtained as:

$$
\dot{\varepsilon}_{i i}^{\text {pore }}=p\left(\frac{1}{\zeta}+\frac{3}{4 \eta}\right)
$$

To obtain $\zeta$, we consider the consistency condition based upon the fact that the macroscopic strain rate is the volume average of the microscopic strain rate on the pore and the matrix phase. In particular, if we consider the trace of the strain rate tensor, one has:

$$
\dot{\varepsilon}_{i i}=\theta \dot{\varepsilon}_{i i}^{\text {pore }}+(1-\theta) \dot{\varepsilon}_{i i}^{\text {matrix }}
$$

Because of the matrix incompressibility, $\dot{\varepsilon}_{i i}^{\text {matrix }}=0$, and we end up with:

$$
\zeta=\frac{4}{3} \eta \frac{1-\theta}{\theta}=\frac{4}{3} \eta_{o} \frac{(1-\theta)^{3}}{\theta}
$$

where the expression (A2) of the effective shear modulus $\eta$ has been used. Comparison with Eqs. (13) provides:

$$
\psi(\theta)=\frac{2}{3} \frac{(1-\theta)^{3}}{\theta}
$$




\section{A.2. Derivation of the expression for the effective Laplace pressure (sintering stress)}

The effective ${ }^{1}$ Laplace pressure (sintering stress) $P_{\mathrm{L}}$ is the result of the collective action of local capillary stresses in a porous material. In this section, we shall determine $P_{\mathrm{L}}$ with the simplest approach considering the sintering stress as a function of porosity only. Below two possible derivations of the expression for the effective Laplace pressure: (i) based upon the stochastic approach employed by Skorohod (1972), and (ii) using averaging of energy dissipation are represented. A variety of other approaches can be found in the literature (Lenel et al., 1961; Gregg and Rhines, 1973; Beere, 1975; Coble, 1978; Bhat and Arunachalam, 1980; DeJonghe and Rahaman, 1984, 1988; Swinkels and Ashby, 1985; Hsueh et al., 1986; Rahaman et al., 1986; Raj, 1987; Bordia and Scherer, 1988; Panda et al., 1989; Ashby, 1990; McMeeking and Kuhn, 1992; Svoboda. et al., 1994; Riedel et al., 1994; Svoboda and Riedel, 1995a,b; Svoboda et al., 1996; Bouvard and McMeeking, 1996).

\section{A.2.1. Derivation of the expression for the sintering stress using a stochastic approach}

As shown before [see Eq. (8)], the effective Laplace pressure $P_{L}$ is obtained by derivation of the free energy per unit mass with respect to the specific volume of the porous material [Eq. (8)]. For sintering, the free energy can be reduced to the free surface energy $F_{\mathrm{S}}$. Therefore, we have:

$$
P_{\mathrm{L}}=\frac{\mathrm{d} F_{\mathrm{S}}}{\mathrm{d} \vartheta}
$$

Here $F_{\mathrm{S}}$ is the free surface energy, and $v$ is the specific volume of porous material, which can be decomposed into:

$$
\vartheta=\vartheta_{p}+\vartheta_{m}
$$

where $v_{p}$ and $v_{m}$ are the parts corresponding to the pores and to the matrix (skeleton), respectively. Because of matrix incompressibilty (A9) can be rewritten as:

$$
P_{\mathrm{L}}=\frac{\mathrm{d} F_{\mathrm{S}}}{\mathrm{d} \vartheta_{p}}
$$

The specific surface area of a porous body is defined by:

$$
\xi=\frac{\Omega}{\vartheta_{m}}
$$

where $\Omega$ is the total free surface area of pores contained in volume $\vartheta$.

\footnotetext{
1 "Effective" sintering stress is associated with the porous material as an effective medium including both substance and voids, while the "local" sintering stress acts at the surface of a pore.
} 
The derivative $\frac{\mathrm{d} \xi}{\mathrm{d} \theta}$ determines the moving force for sintering:

$$
\frac{\mathrm{d} F_{\mathrm{S}}}{\mathrm{d} \vartheta}=\alpha \frac{\mathrm{d} \xi}{\mathrm{d} \theta}(1-\theta)^{2}
$$

where $\alpha$ is a surface tension.

For the derivation of Eq. (A13), the following relationships are taken into consideration:

$$
\frac{\vartheta-\vartheta_{m}}{\vartheta_{m}}=\theta ; \frac{\mathrm{d} F_{\mathrm{S}}}{\mathrm{d} \theta}=\frac{\mathrm{d} F_{\mathrm{S}}}{\mathrm{d} \xi} \cdot \frac{\mathrm{d} \xi}{\mathrm{d} \theta} \cdot \frac{\mathrm{d} \theta}{\mathrm{d} \vartheta} ; \frac{\mathrm{d} F_{\mathrm{S}}}{\mathrm{d} \Omega}=\alpha ; \frac{\mathrm{d} \theta}{\mathrm{d} \vartheta}=\frac{(1-\theta)^{2}}{\vartheta_{m}}
$$

We assume a one to one correspondence between $\xi$ and the porosity $\theta$ as well as with the mean curvature of the pore surface, which can be estimated for porous powder systems as being inversely proportional to the mean radius of powder particles $r_{o}$ :

$$
\xi=\frac{B}{r_{o}} g(\theta)
$$

where $B$ is a constant which is equal to 3 for spherical particles. The function $g(\theta)$ should satisfy certain requirements: it should be monotonously increasing with respect to $\theta$, it should be equal to zero when $\theta=0$ and it should be finite when $\theta \rightarrow 1$. The latter requirement is connected with the fact that the surface of a porous body cannot be larger than the full summarized surface of particles, which is equal to $\frac{B}{r_{o}}$. For an ideal statistical composition of isomeric quasispherical pores and particles, on the basis of an elementary probabilistic analysis, it follows (Skorohod, 1972):

$$
g(\theta)=\theta
$$

Finally, we obtain:

$$
P_{\mathrm{L}}=\frac{3 \alpha}{r_{o}}(1-\theta)^{2}
$$

\section{A.2.2. Derivation of the sintering stress from averaging of the energy dissipation}

Let us consider a pore surrounded by a spherical layer of fully-dense material with the radius of $R_{1}$ (Fig. A1). At the surface of the pore, the pressure $P_{\mathrm{L} 0}$ is applied which is associated with the local sintering stress equal to $\frac{2 \alpha}{r_{0}}$ where $\alpha$ is the surface tension and $r_{0}$ is a characteristic radius (Skorohod, 1972). The porosity is determined as the pore volume fraction: 


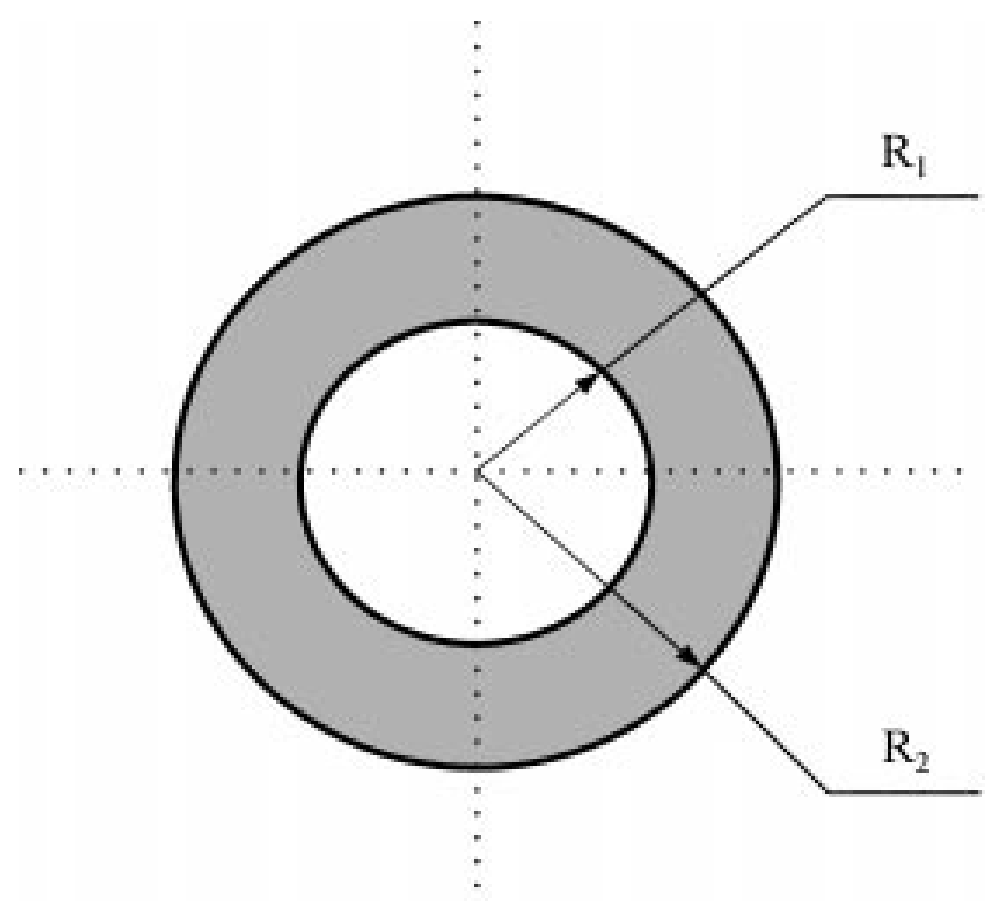

Fig. A1. A representative element of porous medium.

$$
\theta=\left(\frac{R_{1}}{R_{2}}\right)^{3}
$$

The external boundary $\left(r=R_{2}\right)$ is considered to be free of stresses. In the case of linear-viscous properties of the substance, the radial velocities of the material flow are determined as follows:

$$
V_{r}=-\frac{3 P_{\mathrm{L} 0} R_{1}^{3} R_{2}^{3}}{4 \eta_{0}\left(R_{2}^{3}-R_{1}^{3}\right)} \frac{1}{r^{2}}
$$

where $\eta_{0}$ is the shear viscosity modulus.

The radial and the circumferential strain rates are determined as:

$$
\left\{\begin{array}{l}
\dot{\varepsilon}_{r r}=\frac{\partial V_{r}}{\partial r}=\frac{3}{2} \frac{P_{\mathrm{L} 0}}{\eta_{0}} \frac{1}{1-\left(\frac{R_{1}}{R_{2}}\right)^{3}}\left(\frac{R_{1}}{r}\right)^{3} \\
\dot{\varepsilon}_{\phi \phi}=\frac{\partial V_{r}}{\partial r}=\frac{3}{4} \frac{P_{\mathrm{L} 0}}{\eta_{0}} \frac{1}{1-\left(\frac{R_{1}}{R_{2}}\right)^{3}}\left(\frac{R_{1}}{r}\right)^{3}
\end{array}\right.
$$


The second invariant $\gamma_{0}$ of the strain rate deviator (the shape change rate) is given by:

$$
\gamma_{0}=\sqrt{\frac{2}{3}}\left|\dot{\varepsilon}_{r r}-\dot{\varepsilon}_{\phi \phi}\right|=\frac{\sqrt{6}}{4} \frac{P_{\mathrm{L} 0}}{\eta_{0}} \frac{1}{1-\left(\frac{R_{1}}{R_{2}}\right)^{3}}\left(\frac{R_{1}}{r}\right)^{3}
$$

The dissipation of energy per unit volume of matrix is determined as follows:

$$
d=\eta_{0} \gamma_{0}^{2}
$$

Averaging this value over the spherical layer volume, one can obtain:

$$
<d>_{\text {matrix }}=\frac{3 \int_{R_{1}}^{R_{2}} d \cdot r^{2} \mathrm{~d} r}{R_{2}^{3}-R_{1}^{3}}=\frac{3}{8} \frac{P_{\mathrm{L} 0}^{2}}{\eta_{0}} \frac{\left(R_{1} / R_{2}\right)^{3}}{\left[1-\left(\frac{R_{1}}{R_{2}}\right)^{3}\right]^{2}}
$$

Substituting expression for porosity (A18), we have:

$$
<d>_{\text {matrix }}=\frac{3}{8} \frac{P_{\mathrm{L} 0}^{2}}{\eta_{0}} \frac{\theta}{(1-\theta)^{2}}
$$

For the effective porous material, it follows from Eq. (16) that the equivalent effective strain rate $W$ is equal to:

$$
W=\frac{\sqrt{\psi}}{\sqrt{1-\theta}}|e|
$$

Using Eq. (15), the volume dissipation for the effective porous material is given by:

$$
D=(1-\theta) \eta_{0} W^{2}=\eta_{0} \psi \mathrm{e}^{2}
$$

From Eq. (20), for free sintering $(p=0)$, we derive:

$$
e=-\frac{P_{\mathrm{L}}}{2 \eta_{0} \psi}
$$

where $P_{\mathrm{L}}$ is the effective sintering stress.

Substituting Eq. (A27) into Eq. (A26), we obtain:

$$
D=\frac{1}{4} \frac{P_{\mathrm{L}}^{2}}{\eta_{0}} \frac{1}{\psi}
$$


From Hill's identity we have:

$$
D=(1-\theta)<d>_{\text {matrix }}
$$

Using Eqs. (A24), (A28) and (A29), we get:

$$
P_{\mathrm{L}}=P_{\mathrm{L} 0} \sqrt{\frac{3}{2} \psi \frac{\theta}{1-\theta}}
$$

Eq. (A30) can be employed for the determination of the effective sintering stress if the effective bulk viscosity $\psi(\theta)$ is known. Using Eq. (A8), we derive:

$$
P_{\mathrm{L}}=P_{\mathrm{L} 0}(1-\theta)
$$

or:

$$
P_{\mathrm{L}}=\frac{2 \alpha}{r_{o}}(1-\theta)
$$

\section{References}

Ashby, M.F., 1990. Background Reading, HIP 6.0. University of Cambridge, Cambridge, UK.

Beere, W., 1975. The second stage sintering kinetics of powder compacts. Acta Metall. 23 (1), 139-145.

Bhat, T.B., Arunachalam, V.S., 1980. Inhomogeneous flow and the effective pressure concept in pressure sintering. J Mater. Sci. 15, 1614-1618.

Bordia, R.K., Jagota, A., 1993. Crack growth and damage in constrained sintering films. J Amer. Ceram. Soc. 76 (10), 2475.

Bordia, R.K., Scherer, G.W., 1988. Overview no. 70. On constrained sintering I, II, III. Acta Metall. 36 (9), 2393,2399,2422.

Bouvard, D., McMeeking, R.M., 1996. The deformation of interparticle necks by diffusion controlled creep. J Amer. Ceram. Soc. 79, 666-672.

Brett, Seigle, 1963. Shrinkage of voids in copper. Acta Met. 11, 467-474.

Chen, L.Q., Fan, D.A., 1996. Computer simulation model for coupled grain growth and Ostwald ripening-application to $\mathrm{AL}_{2} \mathrm{O}_{3}-\mathrm{ZrO}_{2}$ two-phase systems. J Amer. Ceram. Soc. 79, 1163-1168.

Coble, R.L., 1961. Sintering crystalline solids. 1. Intermediate and final state diffusion models. J Appl. Phys. 32, 787.

Cocks, A.C.F., 1994. Overview no. 117. The structure of constitutive laws for the sintering of fine grained materials. Acta Metall. 42 (7), 2191.

Cocks, A.C.F., Aparicio, N.D., 1995. Diffusional creep and sintering - the application of bounding theorems. Acta Metall. 43 (2), 731.

Cocks, A.C.F., Du, Z.Z., 1993. Pressureless sintering and HIPing of inhomogeneous ceramic compacts. Acta Metall. 41 (7), 2113.

Corapcioglu, Y., Uz, T., 1978. Constitutive equations for plastic deformation of porous materials. J Powd. Tech. 21, 269.

DeJonghe, L.C., Rahaman, M.L., 1984. Pore shrinkage and sintering stress. J Amer. Ceram. Soc. 67, C214-C215. 
DeJonghe, L.C., Raharnan, M.N., 1988. Sintering stress of homogeneous and heterogeneous powder compacts. Acta Metall. 36 (1), 223.

Doraivelu, S.M., Gegel, H.L., Gunasekera, J.S., Malas, J.C., Morgan, J.T., 1984. A new yield function for compressible P/M materials. J Mech. Sci. 26 (9/10), 527-535.

Druyanov, B.A., Vartanov, K.B., 1984. A thermomechanical theory of deformation of porous and powder media incorporating "spontaneous" densification. Sov. Powd. Metall. 263 (11), 29.

Du, Z.-Z., Cocks, A.C.F., 1992a. Constitutive models for the sintering of ceramic components - I. Material Models. Acta Metall. 40 (8), 1969.

Du, Z.-Z., Cocks, A.C.F., 1992b. Constitutive models for the sintering of ceramic components - II. Sintering of inhomogeneous bodies. Acta Metall. 40 (8), 1981.

Fleck, N.A., Kuhn, L.T., McMeeking, R.M., 1992. Yielding of metal powder bonded by isolated contacts. J Mech. Phys. Solids 40 (5), 1139.

German R.M. (1996), Sintering Theory and Practice, John Wiley and Sons, Inc.

Goldsmith, A., Waterman, T.E., Hirschhom, H.J., 1961. Handbook of Thermophysical Properties of Solid Materials. Vol. III: Ceramics, Macmillian Co, New York.

Green, R.G., 1972. A plasticity theory for porous solids. Int. J Mech. Sci. N4, 109-120.

Gregg, R.A., Rhines, F.N., 1973. Surface tension and the sintering force in copper. Metall. Trans. 4 (5), 1365-1374.

Gurson, A.L., 1977. Continuum theory of ductile rupture by void nucleation and growth, part 1. Yield criteria and flow rules for porous ductile media. J Eng. Mater. Techn. 99, 2.

Helle, A.S., Easterling, K.E., Ashby, M.F., 1985. Hot-isostatic pressing diagrams: new developments. Acta Metall. 12, 2163-2174.

Hong, W., Dharani, L.R., 1995. Pressureless sintering of a ceramic-matrix with multiple rigid inclusions finite-element model. J Amer. Ceram. Soc. 78, 1593-1600.

Hsueh, C.H., Evans, A.G., Cannon, R.M., Brook, R.J., 1986. Visco-elastic stresses and sintering damage in heterogeneous powder compacts. Acta Metall. 34, 927.

Jagota, A., Hui, C.Y., 1990a. Mechanics of sintering thin films - I. Formulation and analytical results. Mech. Mater. 1, 221.

Jagota, A., Hui, C.Y., 1990b. Mechanics of sintering thin films - II. Cracking due to self-stress. Mech. Mater. 9, 107.

Kim, K.T., Carroll, M.M., 1987. Compaction equations for strain hardening porous materials. Int. J Plasticity 3, 63-73.

Kingery, W.D., 1967. Introduction to Ceramics. Wiley, New York.

Kuhn, H.A., Downey, C.L., 1971. Deformation characteristics and plasticity theory of sintered powder materials. Int. J Powd. Metall. 7 (1), 15.

Lee, B.J., Mear, M.E., 1992. Constitutive relations for power-law solids containing aligned spheroidal voids. J Mech. Phys. Solids 40, 1805.

Lenel, F.V., Hausner, H.H., Hayashi, E., Ansell, G.S., 1961. The driving force for shrinkage in copper powder compacts during the early stages of sintering. Powder Metall. 8, 186-198.

Mackenzie, J.K., 1950. The elastic constants of a solid containing spherical holes. Proc. Phys. Soc. 63-B, $2-11$.

McMeeking, R.M., 1992. Constitutive laws for sintering and pressing of powders. Mechanics of Granular Materials and Powder Systems. ASME 37, 51.

McMeeking, R.M., Kuhn, L.T., 1992. Diffusional creep law for powder compacts. Acta Mater. 40 (5), 961.

Molinari, A., 1988, Shear band analysis. In: Nonlinear Phenomena in Material Sciences. Solid State Phenomena, Vols. 3-4, Trans. Tech. Publ. pp. 447-468.

Molinari, A., Estrin, Y., Dudzinski, D., 1993. Thermomechanical instability associated with heat release during plastic flow. Int. J Plasticity 9 (3), 317-343.

Morgan, C.S., 1973. Material transport by dislocation motion in sintering. Phys. Sinter. 5, 31-40.

Mori, K., 1992. Finite element simulation of nonuniform shrinkage in sintering of ceramic powder compact. NUMIFORM92, Sophia-Antipolis, Rotterdam, pp. 69-78.

Olevsky, E., 1994. On continuum simulation of consolidation in porous media. Proc. of Powder Metallurgy World Congress, Paris, Vol. 2, p. 697. 
Olevsky, E., Bert, C., 1997. A comparative analysis of viscous sintering problems by the differential quadrature and the finite element methods. Comm. Num. Meth. Eng. 13, 355-372.

Olevsky E., Skorohod V., 1988. Some questions of sintering kinetics under external forces influence. Technological and Design Plasticity of Porous Materials, IPMS NAS Ukraine, p. 97.

Olevsky, E., Skorohod, V., 1993. Deformation aspects of anisotropic-porous bodies sintering. Journal de Physique IV C7 (3), 739.

Olevsky, E, Dudek, H.J., Kaysser, W.A., 1996. HIPing conditions for processing of metal matrix composites using continuum theory for sintering I. Theoretical analysis. Acta Mater. 44 (N2), 707-713.

Olevsky, E., Timmermans, G., Shtern, M., Froyen, L., Delaey, L., 1997. The permeable element method for modeling of deformation processes in porous and powder materials: Theoretical basis and checking by experiments. Powd. Technol. 93/2, 123-141.

Panda, P.C., Mobley, W.M., Raj, R., 1989. Effect of the heating rate on the relative rates of sintering and crystallization in glass. J. Amer. Ceram. Soc. 72 (12), 2361-2364.

Ponte Castaneda, P., 1991. The effective mechanical properties of nonlinear isotropic composites. J. Mech. Phys. Solids 39, 45.

Rahaman, M.N., DeJonghe, L.C., 1990. Sintering of glass powder under a uniaxial stress. J. Amer. Ceram. Soc. 73 (3), 707.

Rahaman, M.N., Brook, R.J., DeJonghe, L.C., 1986. Effect of shear-stress on sintering. J. Amer. Ceram. Soc. $69,53-58$.

Raiser, G., Clifton, R.J., 1993. High strain rate deformation and damage in ceramic materials. J. Eng. Mater. Technol. - Trans. ASME 115, 292-299.

Raj, R., 1987. Analysis of the sintering pressure. J. Amer. Ceram. Soc. 70, C210-C211.

Reid, C.R., 1994. Nui-nerical simulation of free shrinkage using a continuum theory for sintering. Powder Technology 81, 287-291.

Reid, C.R., Oakberg, R.G., 1990. A continuum theory for the mechanical response of materials to the thermodynamic stress of sintering. Mech. Mater. 10, 203-213.

Riedel, H., 1990. A constitutive model for the finite-element simulation of sintering - distortions and stresses. In: Messing, G.L. (Ed.), Ceramic Powder Science III. Amer Ceram Soc, Westerville, OH, pp. 619.

Riedel, H., Svoboda, J., 1993. A theoretical study of grain growth in porous solids during sintering. Acta Metall. 41 (6), 1929.

Riedel, H., Kozak, V., Svoboda, J., 1994. Densification and creep in the final stage of sintering. Acta Metall. 42 (9), 3093.

Scherer, G.W., 1977. Sintering of low-density glasses: I,II,III. J. Amer. Ceram. Soc. 60 (5), 236, 239, 243.

Scherer, G.W., 1979. Sintering of inhomogeneous glasses: Application to optical waveguides. J. NonCryst. Solids 34, 239.

Schatt, W., Friedrich, E., Wieters, K.P., 1986. Dislocation-activated sintering. Rev. Powd. Met. Phys. Ceram. 3, 1-111.

Shima, S., Oyane, M., 1976. Plasticity theory for porous metals. Int. J Mech. Sci. N6, 285-291.

Shtern, M.B., Serdyuk, G.G., Maximenko, L.A., Truhan, Y.V., Shulyakov, Y.M., 1982. Phenomenological Theories of Pressing of Powders. Naukova Durnka, Kiev.

Skorohod, V.V., 1972. Rheological Basis of the Theory of Sintering. Kiev, Naukova Durnka.

Skorohod, V.V., Olevsky, E.A., Shtern, M.B., 1989. Questions of the mathematical modeling of sintering under the external force influence. Proc. of IX Int. Conf on Powder Metallurgy, Dresden, Vol. pp. 43-57.

Skorohod, V., Olevsky, E., Shtern, M., 1991. Continuum theory of sintering of porous bodies: model and application. Science of Sintering 23 (2), 79-91.

Skorohod, V., Olevsky, E., Shtern, M., 1993a. Continuum theory of sintering - I. Phenomenological model. Analysis of the external forces influence on the sintering kinetics. Sov. Powd. Metall. 361 (1), 22.

Skorohod, V., Olevsky, E., Shtern, M., 1993b. Continuum theory of sintering - II. Effect of the rheological properties of the solid phase on the sintering kinetics. Sov. Powd Metall. 362 (2), 16.

Skorohod, V., Olevsky, E., Shtern, M., 1993c. Continuum theory of sintering - III. Effect of the nonhomogeneous distribution of the compact parameters and their fixation conditions on the sintering kinetics. Sov. Powd. Metall 363 (3), 208. 
Skorohod, V.V., Solonin, S.M., 1984. Physical-Metallurgical Basis of Sintering of Powders. Moscow, Metallurgiya.

Sofronis, P., McMeeking, R.M., 1992. Creep of power-law material containing spherical voids. Trans. of ASME 59, 88-95.

Svoboda, J., Riedel, H., 1992. Pore-boundary interactions and evolution equations for the porosity and the grain size during sintering. Acta Metall. 40 (11), 2829.

Svoboda, J., Riedel, H., 1995a. New solutions describing the formation of interparticle necks in solid-state sintering. Acta Metall. 43 (1), 1.

Svoboda, J., Riedel, H., 1995b. Quasi-equilibrium sintering for coupled grain-boundary and surface diffusion. Acta Metall. 43 (2), 499.

Svoboda, L., Riedel, H., Zipse, H., 1994. Equilibrium pore surfaces, sintering stresses and constitutive equations for the intermediate and late stages of sintering, I, II. Acta Mater. 42, 435, 445.

Svoboda, J., Riedel, H., Gaebel, R., 1996. A model for liquid phase sintering. Acta Mater. 44 (8), 3215.

Swinkels, F.B., Ashby, M.F., 1985. A second report on sintering diagrams. Acta Metall. 29, 259-281.

Tvergaard, V., 1982. On localization in ductile materials containing spherical voids. Int. J Fracture 18, $237-252$.

Venkatachari, K.R., Raj, R., 1986. Shear deformation and densification of powder compacts. J. Amer. Ceram. Soc. 69 (6), 499.

Wilkinson, D.S., Ashby, M.F., 1975. Pressure sintering by power law creep. Acta Metall. 23, 1277-1285.

Zhao, Y., Dharani, L.R., 1994. Theoretical model for the analysis of a ceramic thin-film sintering on a non-sintering substrate. Thin Solid Films 245, 109-114. 